This item was submitted to Loughborough's Research Repository by the author.

Items in Figshare are protected by copyright, with all rights reserved, unless otherwise indicated.

\title{
Design of optimal robust fixed-structure controllers using the quantitative feedback theory approach
}

PLEASE CITE THE PUBLISHED VERSION

\section{PUBLISHER}

(C) IMechE / Professional Engineering Publishing

\section{VERSION}

VoR (Version of Record)

\section{LICENCE}

CC BY-NC-ND 4.0

\section{REPOSITORY RECORD}

Halikias, G.D., Argyrios C. Zolotas, and R. Nandakumar. 2019. "Design of Optimal Robust Fixed-structure Controllers Using the Quantitative Feedback Theory Approach”. figshare. https://hdl.handle.net/2134/4520. 
This item was submitted to Loughborough's Institutional Repository (https://dspace.lboro.ac.uk/) by the author and is made available under the following Creative Commons Licence conditions.

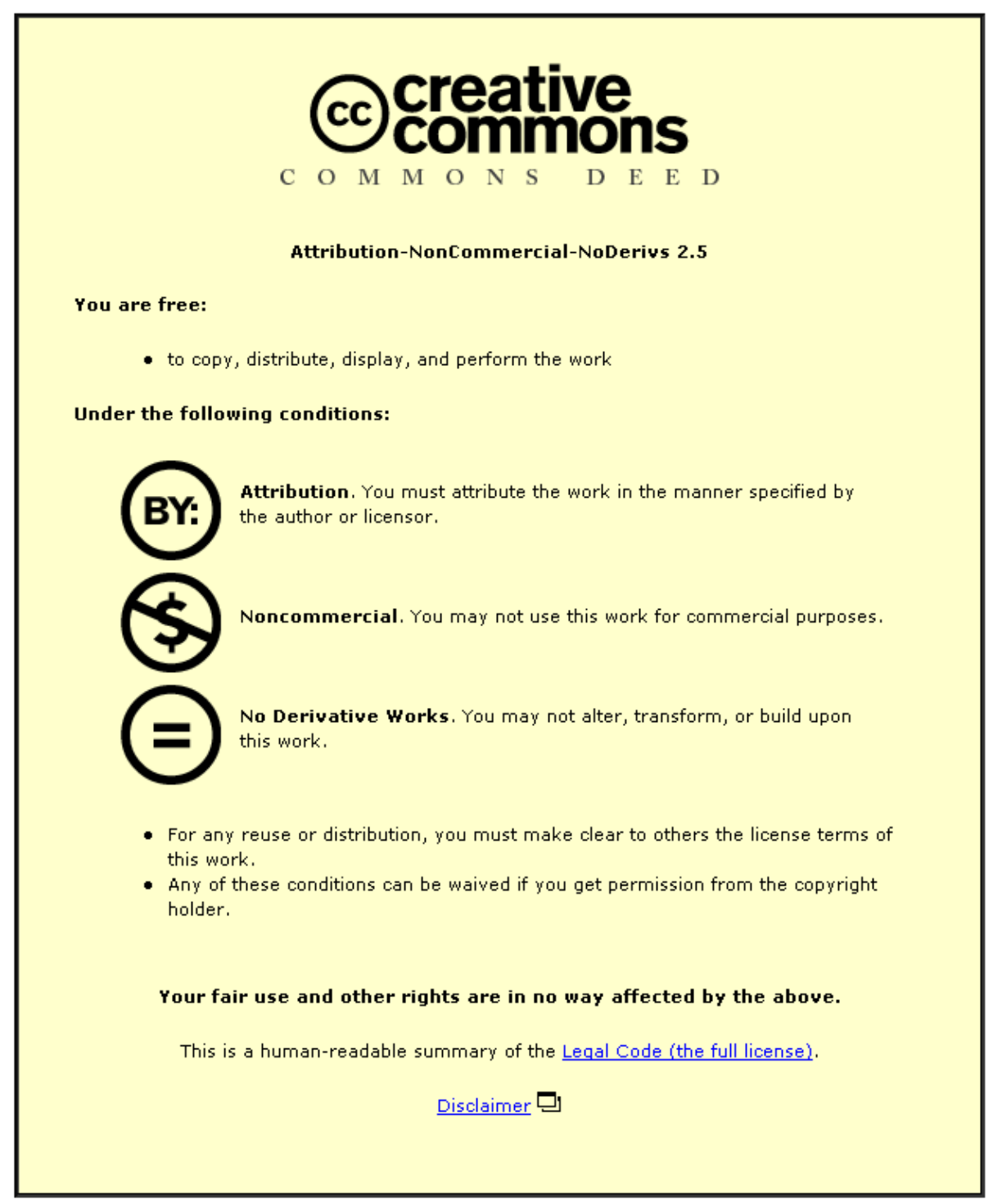

For the full text of this licence, please go to: http://creativecommons.org/licenses/by-nc-nd/2.5/ 


\title{
Design of optimal robust fixed-structure controllers using the quantitative feedback theory approach
}

G D Halikias $^{1}$, A C Zolotas ${ }^{2 *}$, and $R$ Nandakumar ${ }^{1}$

${ }^{1}$ School of Engineering and Mathematical Sciences, City University, London, UK

${ }^{2}$ Department of Electronic and Electrical Engineering, Loughborough University, Loughborough, UK

The manuscript was received on 23 May 2006 and was accepted after revision for publication on 5 February 2007.

DOI: 10.1243/09596518JSCE305

\begin{abstract}
A simple optimization algorithm is proposed for designing fixed-structure controllers for highly uncertain systems. The method can be used to automate the loop-shaping step of the quantitative feedback theory (QFT) design procedure and guarantees robust stability and performance to the feedback loop for all parameters in the plant's uncertainty set. To avoid over-designing the system, the algorithm can be used to minimize either the asymptotic gain, the open-loop crossover frequency or the $3 \mathrm{~dB}$ bandwidth of the closed-loop system (nominal or worst case). The proposed algorithm is illustrated with a design example involving a hydraulic actuator, carried out within a computer aided design (CAD) environment ('StdQFT' toolbox) which has been developed by the authors. Some preliminary results of this work appeared in $2002[1]$.
\end{abstract}

Keywords: robust control, quantitative feedback theory, loop-shaping, optimization, hydraulic actuator

\section{INTRODUCTION}

Quantitative feedback theory (QFT) is a systematic robust control design methodology for systems subject to large parametric or unstructured uncertainty. QFT is a graphical loop-shaping procedure used for the control design of either single input-single output (SISO) or multiple input-multiple output (MIMO) uncertain systems, including the non-linear and time-varying cases [2] traditionally carried out on the Nichols chart. The Nichols chart is a useful tool for reading off closed-loop gain and phase directly from a plot of open-loop logarithmic gain and phase parameterized by frequency. It is widely used in classical control and forms an integral part of the standard QFT control design procedure $[3,4]$. Relative to other robust-control design methodologies, QFT offers a number of advantages, apart from using classical control design techniques. These include: (a) the ability to assess quantitatively the 'cost of

* Corresponding author: Department of Electronic and Electrical Engineering, Loughborough University, Ashby Road, Loughborough LE11 3TU, UK.email: a.zolotas@ieee.org feedback' [5-7], (b) the ability to take into account phase information in the design process (this is ignored in many norm-based approaches, e.g. $\mathscr{H}_{\infty}$ optimal control which is based on singular values), and (c) the ability to provide 'transparency' in the design, i.e. clear trade-off criteria between controller complexity and the feasibility of the design objectives. Note that (c) implies in practice that QFT often results in simple controllers that are easy to implement.

The QFT design procedure is based on the twodegree of freedom feedback configuration shown in Fig. 1. In this diagram $G(p, s)$ denotes the uncertain plant, while $K(s)$ and $F(s)$ denote the feedback compensator and pre-filter respectively that are to be designed. Note that model uncertainty is described by the $r$-parameter vector $\boldsymbol{p} \in \mathscr{P} \subseteq \mathscr{R}^{r}$ taking values in the set $\mathscr{P}$; it is further assumed that $G(\boldsymbol{p}, s)$ has the same number of right half-plane (RHP) poles for all $\boldsymbol{p} \in \mathscr{P}$. Translating the uncertainty into the frequency domain gives rise to the plant's 'uncertainty templates', which are the sets

$$
\mathscr{G}_{\omega}=\{G(\boldsymbol{p}, \mathrm{j} \omega): \boldsymbol{p} \in \mathscr{P}\}
$$




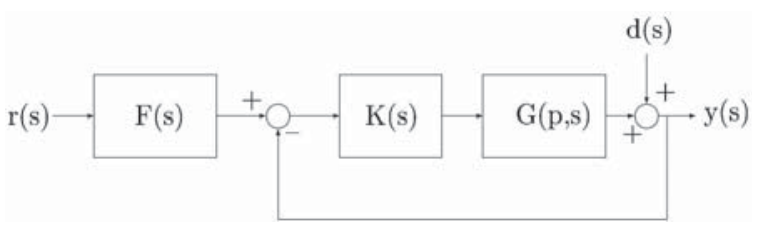

Fig. 1 Feedback configuration

For each fixed frequency $\omega, \mathscr{G}_{\omega}$ defines a 'fuzzy region' on the Nichols chart which describes the uncertainty of the plant at frequency $\omega$ in terms of magnitude (in $\mathrm{dBs}$ ) and phase (in degrees). For design purposes, $N$ uncertainty templates are constructed corresponding to a discrete set of frequencies $\Omega=\left\{\omega_{1}, \omega_{2}, \ldots, \omega_{N}\right\}$, chosen to cover adequately the system's bandwidth.

The robust performance objectives of the design include good tracking of reference input $r(s)$ and good attenuation of the disturbance signal $d(s)$ entering at the system's output, despite the presence of uncertainty. The robust tracking objectives are captured by the set of inequalities

$$
\begin{aligned}
& \max _{\boldsymbol{p} \in \mathscr{P}} \Delta\left|\frac{G\left(\boldsymbol{p}, \mathrm{j} \omega_{i}\right) K\left(\mathrm{j} \omega_{i}\right)}{1+G\left(\boldsymbol{p}, \mathrm{j} \omega_{i}\right) K\left(\mathrm{j} \omega_{i}\right)}\right|_{\mathrm{dB}} \\
& \quad \leqslant \delta\left(\omega_{i}\right):=\left.B_{u}\left(\omega_{i}\right)\right|_{\mathrm{dB}}-\left.B_{l}\left(\omega_{i}\right)\right|_{\mathrm{dB}}
\end{aligned}
$$

for each $i=1,2, \ldots, N$, i.e. if, for each frequency $\omega_{i}$, the maximum variation in the closed-loop gain as $\boldsymbol{p} \in \mathscr{P}$ does not exceed the maximum allowable spread in specifications $\delta\left(\omega_{i}\right)$, typically specified via two appropriate magnitude frequency responses $B_{u}(\omega)=\left|B_{u}(\mathrm{j} \omega)\right|$ and $B_{l}(\omega)=\left|B_{l}(\mathrm{j} \omega)\right|$. Note that it is not necessary to bound the actual gain (but only the gain spread) since it is assumed that: (a) no uncertainty is associated with the feedback controller $K(s)$ and (b) the pre-filter $F(s)$ can provide arbitrary scaling to the closed-loop gain at every frequency.

The robust disturbance-rejection objective is satisfied by bounding the sensitivity function, i.e. by imposing constraints of the form

$$
\max _{\boldsymbol{p} \in \mathscr{P}}\left|\frac{1}{1+G\left(\boldsymbol{p}, \mathrm{j} \omega_{i}\right) K\left(\mathrm{j} \omega_{i}\right)}\right| \leqslant D\left(\omega_{i}\right)
$$

for a (subset) of the design frequencies $\left\{\omega_{1}, \omega_{2}, \ldots, \omega_{N}\right\}$ (normally in the low-frequency range). Again these are typically specified via an appropriate magnitude frequency response $D(\omega)=|D(\mathrm{j} \omega)|$.

Robust stability is enforced by ensuring that: (a) no unstable pole-zero cancellations occur between the plant and the controller (for every $\boldsymbol{p} \in \mathscr{P}$ ), (b) the nominal open-loop frequency response $L_{\mathrm{o}}(\mathrm{j} \omega)=$ $G\left(p_{\mathrm{o}}, \mathrm{j} \omega\right) K(\mathrm{j} \omega)$ (defined for any $\left.\boldsymbol{p}_{\mathrm{o}} \in \mathscr{P}\right)$ does not cross the -1 point, i.e. the $\left(-180^{\circ}, 0\right)$ point on the Nichols chart), and makes a total number of (anticlockwise) encirclements around it equal to the number of unstable poles of $L_{\mathrm{o}}(s)=G\left(\boldsymbol{p}_{\mathrm{o}}, s\right) K(s)$, and (c) that no (perturbed) open-loop response crosses the -1 point, i.e.

$$
-1 \notin \bigcup_{\omega \in \mathscr{R}} K(\mathrm{j} \omega) \mathscr{G}_{\omega}
$$

Note that condition (a) is automatically satisfied if $K(s)$ is restricted to be stable and minimum phase, while conditions (b) and (c) can be easily tested graphically $[\mathbf{8}, 9]$. In practice, a more severe condition than (c) is imposed: to establish a minimum amount of damping, it is required that the nominal open-loop frequency response does not penetrate a closed contour in the Nichols chart (universal high-frequency $\mathscr{U}$-contour). This is constructed from an appropriate $M$-circle and information about high-frequency gain uncertainty of the plant [6]. Formulation of robust stability via the $\mathscr{U}$-contour assumes that at high frequencies the phase-uncertainty spread of the system is minimal, an assumption that is reasonable for most systems subject to parametric model uncertainty. If this assumption fails (or if model uncertainty is in part unstructured) the $\mathscr{U}$-contour must be replaced by a set of frequencydependent closed templates containing the critical point. This does not affect significantly the proposed method, although for simplicity it is assumed that robust-stability specifications can be formulated via the $\mathscr{U}$-contour.

The robust tracking and disturbance rejection objectives have been formulated as gain inequalities of the closed-loop transfer functions (sensitivity and complementary sensitivity) at the design frequencies. For the purposes of QFT design, these inequalities must be translated into constraints on the nominal open-loop response $L_{\mathrm{o}}(\mathrm{j} \omega)$. This procedure results in a number of contours ('Horowitz tracking templates' $f_{i}^{\mathrm{t}}(\phi)$ and 'Horowitz disturbancerejection templates' $\left.f_{i}^{\mathrm{d}}(\phi)\right)$ for each frequency $\omega_{i}$, $i=1,2, \ldots, N$. These are functions of the phase variable $\phi \in\left(-360^{\circ}, 0^{\circ}\right]$. Thus, robust tracking is satisfied at frequency $\omega_{i}$ if and only if $\left|L_{\mathrm{o}}\left(\mathrm{j} \omega_{i}\right)\right|_{\mathrm{dB}} \geqslant f_{i}^{\mathrm{t}}\left(\phi_{i}\right)$ where $\arg L_{\mathrm{o}}\left(\mathrm{j} \omega_{i}\right)=\phi_{i}$; similarly, robust disturbance rejection is attained at frequency $\omega_{i}$ if and only if $\left|L_{\mathrm{o}}\left(\mathrm{j} \omega_{i}\right)\right|_{\mathrm{dB}} \geqslant$ $f_{i}^{\mathrm{d}}(\phi)$. The robust-performance templates (Horowitz tracking and disturbance rejection) can be easily constructed (within an arbitrary gain tolerance and for a discretized phase grid) using a simple bisection algorithm. This method uses the uncertainty templates of the plant defined at the design frequencies, normally obtained by 'gridding' the uncertainty parameters. This can be computationally expensive 
although more sophisticated methods have been proposed (e.g. references [10] and [11]), and advances in computational power continuously extend the class of practical problems that can be addressed by QFT.

In conclusion, assuming that the condition prohibiting unstable pole/zero cancellations between the plant and the controller is independently verified, the following conditions guarantee robust stability and performance.

1. The winding number of the nominal open-loop system $L_{\mathrm{o}}(\mathrm{j} \omega)$ around the -1 point is equal to the number of RHP poles of $L_{\mathrm{o}}(s)$.

2. The nominal open-loop frequency response $L_{\mathrm{o}}(\mathrm{j} \omega)$ does not penetrate the $\mathscr{U}$-contour.

3. The following inequalities are satisfied for all $i=1,2, \ldots, n$

$$
\left|L_{\mathrm{o}}\left(\mathrm{j} \omega_{i}\right)\right|_{\mathrm{dB}} \geqslant f_{i}^{\mathrm{t}}\left(\phi_{i}\right)
$$

and

$$
\left|L_{\mathrm{o}}\left(\mathrm{j} \omega_{i}\right)\right|_{\mathrm{dB}} \geqslant f_{i}^{\mathrm{d}}\left(\phi_{i}\right)
$$

in which $\phi_{i}=\arg L_{\mathrm{o}}\left(\mathrm{j} \omega_{i}\right)$. These inequalities correspond to the robust tracking and robust disturbance-rejection specifications respectively.

The paper presents a novel algorithm for designing fixed-structure controllers that satisfy the QFT constraints and minimize a measure of system 'over-design' (asymptotic gain, crossover frequency, closed-loop bandwidth). In section 2 the QFT constraints are formulated in the form of a feasibility programme. Section 3 outlines an optimization algorithm which can be used to design simple fixedstructure controllers proportional-integral-derivative (PID), phase lead/lag, second order) in the QFT framework. The algorithm is illustrated in section 3 with a design example in section 4 involving robust force control of a hydraulic actuator. Finally, the main conclusions of the work appear in section 5 .

\section{FORMULATION OF QFT CONSTRAINTS}

In this section the QFT robust stability and performance constraints are first formulated as a feasibility programme. This leads to an optimization algorithm for carrying out optimal QFT designs using a family of simple fixed-structure compensators. This is in contrast to other approaches (e.g. reference [12]), which optimize the open-loop response of the system in the frequency domain and subsequently fit a (potentially high-order) compensator.
As shown in the last section, the QFT robuststability and performance objectives can be translated to graphical constraints on the Nichols chart. The constraints associated with robust performance ('Horowitz tracking' and 'Horowitz disturbancerejection' templates) correspond to open contours; i.e. they split the Nichols chart into two regions (for each design frequency), the high- and low-gain regions. To meet the tracking or disturbance-rejection objective, each nominal open-loop frequency-response point $L_{\mathrm{o}}\left(\mathrm{j} \omega_{i}\right)$ must be placed on the high-gain region of the contour, i.e. forced to satisfy the inequality $\left|L_{\mathrm{o}}\left(\mathrm{j} \omega_{i}\right)\right|_{\mathrm{dB}} \geqslant f_{i}^{\mathrm{t}}\left(\phi_{i}\right) \quad$ (tracking) or $\left|L_{\mathrm{o}}\left(\mathrm{j} \omega_{i}\right)\right|_{\mathrm{dB}} \geqslant f_{i}^{\mathrm{d}}\left(\phi_{i}\right)$ (disturbance rejection), where $\arg L_{\mathrm{o}}\left(\mathrm{j} \omega_{i}\right)=\phi_{i}$. In contrast, the robust-stability template ( $\mathscr{U}$-contour) is a closed contour containing the critical point. To construct the $\mathscr{U}$-contour, a start is made from the definition of an $M$-circle $(M>1)$ in the Nyquist plane $(u, v)$, where $u=\operatorname{Re}\left[L_{\mathrm{o}}(\mathrm{j} \omega)\right]$ and $v=\operatorname{Re}\left[L_{\mathrm{o}}(\mathrm{j} \omega)\right]$

$$
\left|\frac{u+\mathrm{j} v}{1+u+\mathrm{j} v}\right|=M \Rightarrow\left(u+\frac{M^{2}}{M^{2}-1}\right)^{2}+v^{2}=\frac{M^{2}}{\left(M^{2}-1\right)^{2}}
$$

This is a circle of centre

$$
(u, v)=\left(-\frac{M^{2}}{M^{2}-1}, 0\right)
$$

and radius

$$
R=\frac{M}{M^{2}-1}
$$

Since in this case $(M>1)$ the $M$-circle does not contain the origin, it is clear that in the Nichols chart it is defined only for an interval of phases, and is symmetric around the phase line $\phi=-180^{\circ}$. In fact, drawing the tangents to the circle from the origin, it is clear that (see Fig. 2)

$$
\psi_{\max }=\sin ^{-1}\left(\frac{1}{M}\right)
$$

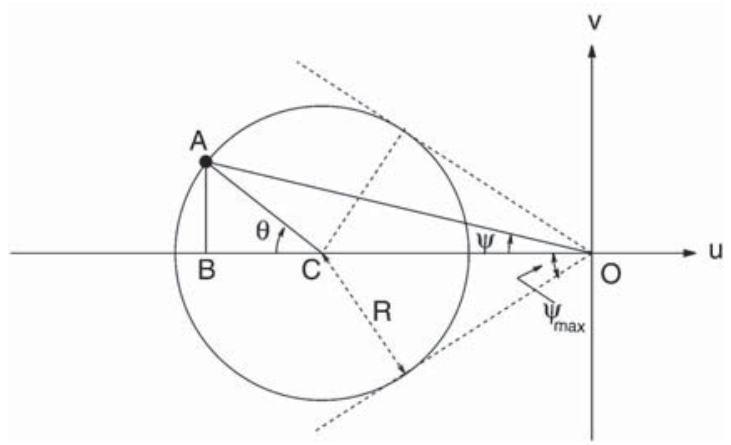

Fig. $2 M$-circle in the Nyquist plane $(M>1)$ 
and hence the $M$-circle is defined on the Nichols chart only for the phase interval

$$
-180^{\circ}-\sin ^{-1}\left(\frac{1}{M}\right) \leqslant \phi \leqslant-180^{\circ}+\sin ^{-1}\left(\frac{1}{M}\right)
$$

Next an equation needs to be derived of the $M$-circle in terms of magnitude $(m)$ and phase $(\phi)$, where

$$
m=\sqrt{u^{2}+v^{2}} \quad \text { and } \quad \phi=\arctan \frac{v}{u}
$$

Referring to Fig. 2, it follows by simple geometry that

$$
m \cos \psi=\frac{M^{2}}{M^{2}-1}+\frac{M}{M^{2}-1} \cos \theta
$$

and

$$
m \sin \psi=\frac{M}{M^{2}-1} \sin \theta
$$

Eliminating variable $\theta$ using the trigonometric identity $\sin ^{2} \theta+\cos ^{2} \theta=1$ results in the quadratic equation

$$
m^{2}-\frac{2 M^{2} \cos \psi}{M^{2}-1} m+\frac{M^{2}}{M^{2}-1}=0
$$

which can be solved as

$$
m=\frac{M^{2}}{M^{2}-1}\left(\cos \psi \pm \sqrt{\frac{1}{M_{2}}-\sin ^{2} \psi}\right)
$$

Thus, using the substitution $\phi=-180^{\circ}-\psi$, the $M$ circle in the Nichols chart is a closed contour which may be decomposed into the union of the graphs of the two functions

$$
\begin{aligned}
M^{+}(\phi)= & 20 \log _{10}\left(-\cos \phi+\sqrt{\frac{1}{M^{2}}-\sin ^{2} \phi}\right) \\
& +20 \log _{10}\left(\frac{M^{2}}{M^{2}-1}\right)
\end{aligned}
$$

and

$$
\begin{aligned}
M^{-}(\phi)= & 20 \log _{10}\left(-\cos \phi-\sqrt{\frac{1}{M^{2}}-\sin ^{2} \phi}\right) \\
& +20 \log _{10}\left(\frac{M^{2}}{M^{2}-1}\right)
\end{aligned}
$$

Following reference [6], the $\mathscr{U}$-contour is obtained by translating $M^{-}(\phi)$ vertically by $V_{\infty} \mathrm{dBs}$, where

$$
V_{\infty}=\lim _{\omega \rightarrow \infty}\left(\max _{\boldsymbol{p} \in \mathscr{P}}|G(\boldsymbol{p}, \mathrm{j} \omega)|_{\mathrm{dB}}-\left|G\left(\boldsymbol{p}_{\mathrm{o}}, \mathrm{j} \omega\right)\right|_{\mathrm{dB}}\right)
$$

This gives the $\mathscr{U}$-contour as the union of the graphs of the two functions

$$
\mathscr{U}^{+}(\phi)=M^{+}(\phi) \quad \text { and } \quad \mathscr{U}^{-}(\phi)=M^{-}(\phi)-V_{\infty}
$$

over the phase interval

$$
\begin{aligned}
\phi_{l} & :=-180^{\circ}-\sin ^{-1}\left(\frac{1}{M}\right) \\
& \leqslant \phi \leqslant-180^{\circ}+\sin ^{-1}\left(\frac{1}{M}\right):=\phi_{h}
\end{aligned}
$$

The ultimate objective of this section is to characterize the regions of the Nichols chart in which the open-loop frequency response point $L_{\mathrm{o}}\left(\mathrm{j} \omega_{i}\right)$ can lie in order to satisfy the robust stability and performance constraints. To this purpose define the composite function as

$$
f_{i}^{\mathrm{m}}(\phi)=\max \left\{f_{i}^{\mathrm{t}}(\phi), f_{i}^{\mathrm{d}}(\phi)\right\}
$$

where the maximum is taken pointwise in $\phi \in$ $\left(-360^{\circ}, 0^{\circ}\right]$. Further define

$$
f_{i}(\phi)= \begin{cases}f_{i}^{\mathrm{m}}(\phi) & \text { for } \phi \leqslant \phi_{l} \\ \max \left\{f_{i}^{\mathrm{m}}(\phi), \mathscr{U}^{+}(\phi)\right\} & \text { for } \phi_{l}<\phi<\phi_{h} \\ f_{i}^{\mathrm{m}}(\phi) & \text { for } \phi \geqslant \phi_{h}\end{cases}
$$

Also let $\Phi_{i}=\left\{\phi: \phi_{l}<\phi<\phi_{h}, f_{i}^{\mathrm{m}}(\phi) \leqslant \mathscr{U}^{-}(\phi)\right\}$. Then, the robust stability and performance constraints at frequency $\omega_{i}$ are satisfied if and only if $L_{\mathrm{o}}\left(\mathrm{j} \omega_{i}\right) \in$ $R_{i} \cup S_{i}$, where

$$
R_{i}=\left\{L_{\mathrm{o}}\left(\mathrm{j} \omega_{i}\right):\left|L_{\mathrm{o}}\left(\mathrm{j} \omega_{i}\right)\right|_{\mathrm{dB}} \geqslant f_{i}(\phi), \phi=\arg L_{\mathrm{o}}\left(\mathrm{j} \omega_{i}\right)\right\}
$$

and

$$
\begin{gathered}
S_{i}=\left\{L_{\mathrm{o}}\left(\mathrm{j} \omega_{i}\right): f_{i}(\phi) \leqslant\left|L_{\mathrm{o}}\left(\mathrm{j} \omega_{i}\right)\right|_{\mathrm{dB}} \leqslant \mathscr{U}^{-}(\phi),\right. \\
\left.\phi=\arg L_{\mathrm{o}}\left(\mathrm{j} \omega_{i}\right) \in \Phi_{i}\right\}
\end{gathered}
$$

An illustration of the region $R_{i} \cup S_{i}$ is given in Fig. 3 . Note that, in practice, when a performance constraint is active, then typically $\Phi_{i}=S_{i}=\varnothing$. This is because performance objectives are normally specified at low frequencies, rarely exceeding the closed-loop bandwidth of the system. However, this formulation allows 'unconstrained' design frequencies to be taken into account, i.e. frequencies at which no performance inequalities apply. Such a frequency $\omega_{i}$ gives $f_{i}^{\mathrm{m}}(\phi)=-\infty$ and hence $\Phi_{i}=\left(\phi_{l}, \phi_{h}\right), \quad S_{i}=\left\{L_{\mathrm{o}} \mathrm{j}\left(\omega_{i}\right)\right.$ : $\left.\left|L_{\mathrm{o}}\left(\mathrm{j} \omega_{i}\right)\right|_{\mathrm{dB}} \leqslant \mathscr{U}^{-}(\phi), \phi=\arg L_{\mathrm{o}}\left(\mathrm{j} \omega_{i}\right) \in\left(\phi_{l}, \phi_{h}\right)\right\}$ (i.e. the region below the $\mathscr{U}$-contour), while $R_{i} \cup S_{i}$ would represent the region outside the $\mathscr{U}$-contour.

The conditions that guarantee the robust-stability and robust-performance specifications at the design 


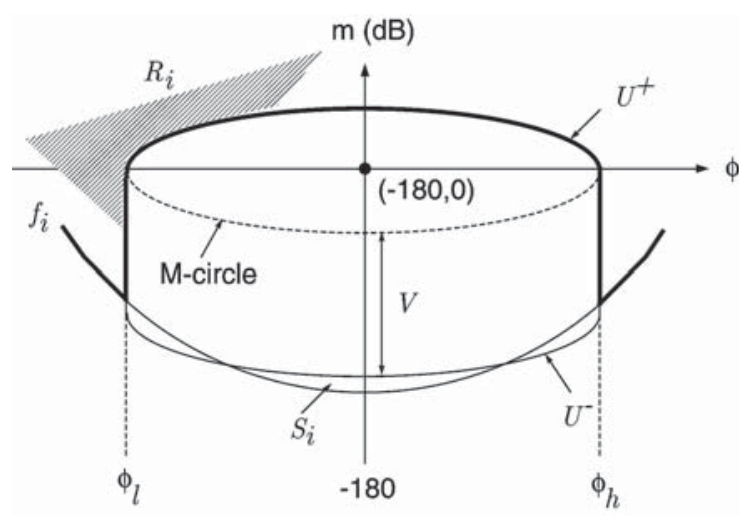

Fig. $3 M$-circle in the Nichols chart $(M>1)$

frequencies can now be summarized by the following two graphical tests.

1. The winding number of the nominal open-loop system $L_{\mathrm{o}}(\mathrm{j} \omega)$ around the -1 point is equal to the number of RHP poles of $L_{\mathrm{o}}(s)$.

2. For each frequency $\omega_{i}, L_{\mathrm{o}}\left(\mathrm{j} \omega_{i}\right) \in R_{i} \cup S_{i}$.

Again, it is assumed that no unstable pole/zero cancellations occur between the controller and $G(\boldsymbol{p}, s)$ for every $\boldsymbol{p} \in \mathscr{P}$, a condition that must be checked independently. Of course, similarly to any QFT-based method, these tests do not really guarantee that $L_{\mathrm{o}}(\mathrm{j} \omega)$ does not enter the $\mathscr{U}$-contour at frequencies other than the design frequencies. This, however, does not cause a problem in practice, provided a reasonably large set of design frequencies is selected near crossover or, alternatively, by slightly tightening the specifications by means of an appropriate tolerance.

\section{OPTIMIZATION ALGORITHM}

In this section an optimization algorithm is outlined for designing fixed-structure compensators of certain types subject to the QFT constraints developed earlier. Every design (i.e. loop shaping of $L_{\mathrm{o}}(\mathrm{j} \omega)$ ) that satisfies the two graphical tests of the last section is in principle 'admissible', i.e. satisfies the robust-stability and robust-performance objectives. Since in general many different designs may be admissible, a method is required for classifying them by formulating an appropriate optimization criterion. Adopting the arguments of Horowitz and Sidi [6, 7], such a criterion must penalize the 'over-design' of the system, e.g. an unnecessarily high closed-loop bandwidth, since this increases the 'cost of feedback' in terms of sensornoise amplification and potential instability due to high-frequency unmodelled dynamics/parasitics.
Appropriate 'cost functions' to be minimized include the following quantities:

(a) open-loop crossover frequency (nominal or worst case);

(b) closed-loop bandwidth (nominal or worst case);

(c) asymptotic open-loop gain;

(d) A measure of the excess gain-bandwidth area which can be expressed as the integral

$$
A\left(\omega_{1}, \omega_{2}\right)=\int_{\omega_{1}}^{\omega_{2}} \log |K(\mathrm{j} \omega)| \mathrm{d} \omega
$$

where $\left[\omega_{1}, \omega_{2}\right]$ is an appropriate frequency interval $[13,14]$.

Each of the above measures can be calculated in a straightforward manner from the frequency response of the system. For example, the open-loop crossover frequency corresponds to the point where the openloop frequency response crosses the $0 \mathrm{~dB}$ line on the Nichols chart. The closed-loop bandwidth is the frequency where the closed-loop gain of the system is $1 / \sqrt{2}(-3 \mathrm{~dB}$ approximately). To calculate the closed-loop bandwidth graphically let $L=r \exp \mathrm{j} \phi$ be the open loop response and set

$$
\frac{|L|}{|1+L|}=\frac{1}{\sqrt{2}} \Rightarrow \frac{r^{2}}{1+r^{2}+2 r \cos \phi}=\frac{1}{2}
$$

This leads to the quadratic equation $r^{2}-2 r \cos \phi-$ $1=0$ whose only admissible solution is $r=\cos \phi+$ $\sqrt{\cos ^{2} \phi+1}$. Thus the closed-loop bandwidth of the system is the frequency at which the open-loop frequency response crosses the curve

$$
N(\phi)=20 \log _{10}\left(\cos \phi+\sqrt{\cos ^{2} \phi+1}\right)
$$

on the Nichols chart, where $\phi$ denotes open-loop phase. The curve $N(\phi)$ is plotted in Fig. 4 over the phase interval $\left(-360^{\circ}, 0^{\circ}\right]$. Finally, the excess gainbandwidth measure $A\left(\omega_{1}, \omega_{2}\right)$ may be easily calculated by numerical integration in terms of the controller parameters.

Note that the open-loop response of most systems encountered in practice crosses the $0 \mathrm{~dB}$ line (or curve $N(\phi)$ ) only once. An important exception consists of systems with lightly damped modes (e.g. flexible structures) exhibiting multiple 'resonance' peaks. In such cases the crossover frequency (or closed-loop bandwidth) is simply defined as the lowest frequency at which crossing occurs. The 'worst-case' crossover frequency or closed-loop bandwidth is defined as the largest frequency among all uncertain frequency responses (contained in the uncertainty template set) crossing the $0 \mathrm{~dB}$ line or curve $N(\phi)$ respectively. Note that all these optimization measures 


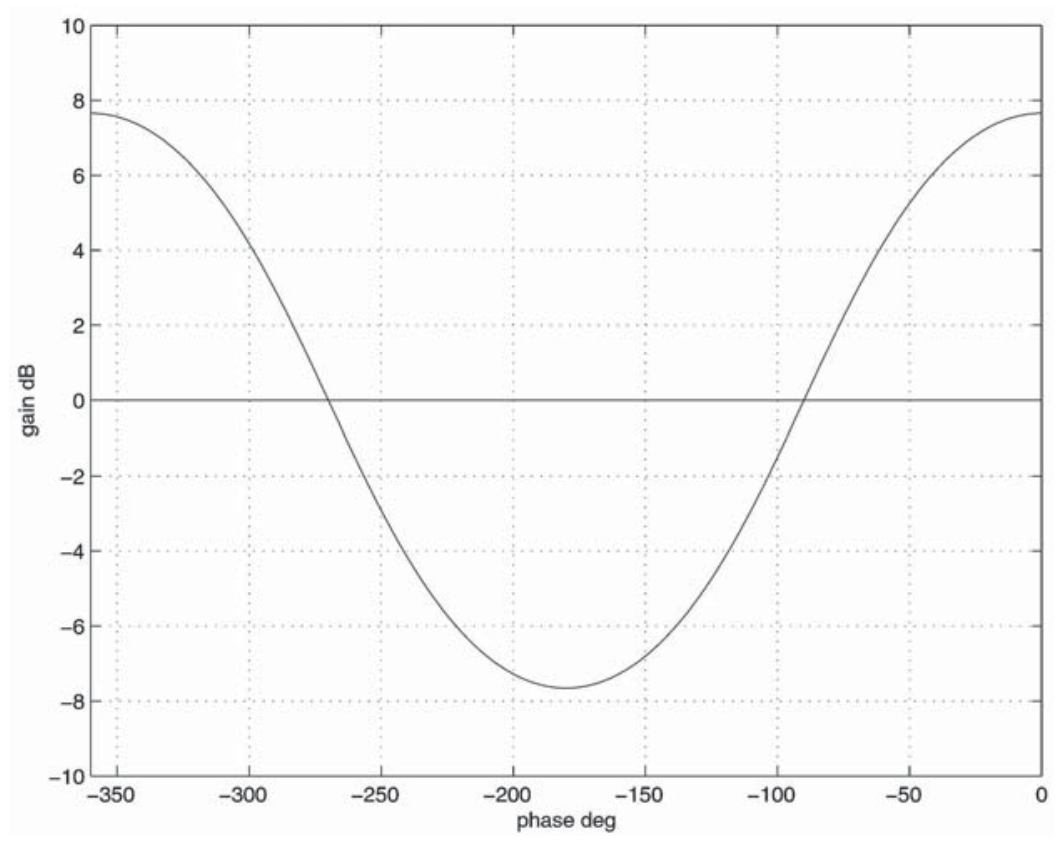

Fig. 4 Curve $N(\phi)$ used to calculate the closed-loop bandwidth

can be easily calculated from the frequency response of the system, possibly using interpolation techniques if high accuracy is required.

The algorithm presented here generalizes previous results $[\mathbf{1}, \mathbf{1 5}]$ and may be used to automate the loopshaping step of the QFT design algorithm (at least partially). This is the most demanding step of the QFT design procedure [16], for which significant research effort has been devoted in the recent literature, e.g. the approach of reference [17] based on Youla's-parametrization and linear programming, the approach of references [18] and [19] which extends the results of references $[\mathbf{2 0}]$ and $[\mathbf{2 1}]$ to the robust QFT framework, techniques that rely on Bode's gainphase integral to impose controller realizability constraints $[12, \mathbf{1 3}, \mathbf{2 2}]$, global optimization of PID controllers using Horowitz bounds [23], etc. Note that these methods are different to those proposed in this paper, which address loop-shaping in an open-loop framework using fixed structure controllers.

The types of compensators considered in this paper are listed below. Note that some of these must be used under appropriate relative-degree assumptions satisfied by the transfer function of the plant.

1. PID: $K(s)=k_{\mathrm{p}}+k_{\mathrm{d}} s+k_{\mathrm{i}} / s$ and $\mathrm{PDD}^{2}: \quad K(s)=$ $k_{1}+k_{2} s+k_{3} s^{2}$

2. First-order lead/lag: $K(s)=k(s+b) / s+a$

3. Second order with complex-poles (zeros): $K(s)=$ $1 / s^{2}+2 \zeta \omega_{n} s+\omega_{n}^{2}\left(\right.$ or $\left.s^{2}+2 \zeta \omega_{n} s+\omega_{n}^{2}\right)$

PID [23] and phase-lead/lag controllers are widely used in industry because they are simple and easy to tune. Thus optimal controllers of the first two types may provide simple solutions to robust control designs based on the QFT method. Note also that every rational controller of arbitrary complexity can be constructed from cascade interconnections of controllers in (2) and (3) above. Thus, it is possible to improve the design continuously by building higher-order controllers in a step-by-step procedure. At each step the optimization algorithm is carried out (for one of the three controller structures) and the resulting optimal controller $K(s)$ is accumulated into the nominal open-loop system by redefining $L_{\mathrm{o}}(s) \leftarrow L_{\mathrm{o}}(s) K(s)$. This process may continue until a satisfactory design is obtained, or until the cost fails to decrease significantly. Of course, the controller resulting from this procedure will not, in general, be optimal over the higher-order controller set.

The proposed algorithm is based on the fact that fixing the phase of the compensator at two distinct frequencies determines the compensator uniquely $u p$ to scaling. Thus, the phase response of the nominal open-loop system is also completely determined, and only a simple calculation is needed to determine the minimum amount of gain required to meet the QFT robust stability and performance specifications (if these are feasible). Geometrically, this corresponds to shifting the frequency response of $L_{\mathrm{o}}(s)$ vertically in the Nichols chart by the minimum gain required to place the points $L_{\mathrm{o}}\left(\mathrm{j} \omega_{i}\right)$ in the $R_{i} \cup S_{i}$ regions while simultaneously satisfying the Nyquist stability encirclement criterion. Repeating this procedure for all possible phase combinations (suitably discretized) 
will eventually produce the optimal design (if one exists). Next, each controller type is considered in turn.

\subsection{PID and PDD ${ }^{2}$ controllers}

The classical PID controller is considered first and is specified by three parameters $k_{\mathrm{i}}, k_{\mathrm{d}}$, and $k_{\mathrm{p}}$ corresponding to the integral, derivative and proportional gains respectively.

\section{Theorem 1}

(a) Let $K(s)=k_{\mathrm{p}}+k_{\mathrm{d}} s+k_{\mathrm{i}} / s$ with $k_{\mathrm{p}}, \quad k_{\mathrm{d}}$, and $k_{\mathrm{i}}$ real parameters. Suppose that $\arg K\left(\mathrm{j} \omega_{i}\right)=\psi_{i}$ and $\arg K\left(\mathrm{j} \omega_{j}\right)=\psi_{j}$, where $\omega_{i} \neq \omega_{j}$. Then the matrix

$$
\mathbf{A}_{i j}=\left(\begin{array}{ccc}
1 & -\frac{1}{\omega_{i}^{2}} & -\frac{\tan \left(\psi_{i}\right)}{\omega_{i}} \\
1 & -\frac{1}{\omega_{j}^{2}} & -\frac{\tan \left(\psi_{j}\right)}{\omega_{j}}
\end{array}\right)
$$

has full (row) rank. Let $\left(V^{i j}\right)=\left[\begin{array}{lll}V_{1}^{i j} & V_{2}^{i j} & V_{3}^{i j}\end{array}\right]^{\prime} \in \mathscr{R}^{3}$ be a (real) non-zero vector in the (one-dimensional) kernel of $\mathbf{A}_{i j}$. Then

$$
\left(\begin{array}{c}
k_{\mathrm{d}} \\
k_{\mathrm{i}} \\
k_{\mathrm{p}}
\end{array}\right)=\lambda\left(\begin{array}{c}
V_{1}^{i j} \\
V_{2}^{i j} \\
V_{3}^{i j}
\end{array}\right):=\lambda\left(\begin{array}{c}
\frac{\omega_{i} \tan \psi_{i}-\omega_{j} \tan \psi_{j}}{\omega_{i}^{2}-\omega_{j}^{2}} \\
\frac{\omega_{i} \omega_{j}\left(\omega_{j} \tan \psi_{i}-\omega_{i} \tan \psi_{j}\right)}{\omega_{1}^{2}-\omega_{j}^{2}} \\
1
\end{array}\right)
$$

where $\lambda$ is an arbitrary real constant. Moreover, the gain and phase of the controller at any frequency is $\omega$ given by

$$
\begin{aligned}
& |K(\mathrm{j} \omega)|=|\lambda| \sqrt{\begin{array}{l}
1+\left[\frac{\omega\left(\omega_{i} \tan \psi_{i}-\omega_{i} \tan \psi_{j}\right)}{\omega_{i}^{2}-\omega_{j}^{2}}\right. \\
\left.-\frac{\omega_{i} \omega_{j}\left(\omega_{j} \tan \psi_{i}-\omega_{i} \tan \psi_{j}\right)}{\omega\left(\omega_{i}^{2}-\omega_{j}^{2}\right)}\right]^{2}
\end{array}} \\
& :=|\lambda| C_{i j}(\omega)
\end{aligned}
$$

and

$$
\arg K(\mathrm{j} \omega)
$$

$$
\begin{aligned}
:=\psi(\omega)=\arctan [ & \frac{\omega\left(\omega_{i} \tan \psi_{i}-\omega_{j} \tan \psi_{j}\right)}{\omega_{i}^{2}-\omega_{j}^{2}} \\
& \left.-\frac{\omega_{i} \omega_{j}\left(\omega_{j} \tan \psi_{i}-\omega_{i} \tan \psi_{j}\right)}{\omega\left(\omega_{i}^{2}-\omega_{j}^{2}\right)}\right]
\end{aligned}
$$

respectively. (b) If the controller gains $k_{\mathrm{p}}, k_{\mathrm{i}}$, and $k_{\mathrm{d}}$ are restricted to be non-negative, then the constraints $\arg K\left(\mathrm{j} \omega_{i}\right)=$ $\psi_{i}$ and $\arg K\left(\mathrm{j} \omega_{j}\right)=\psi_{j}$ are feasible if the two scalars $V_{1}^{i j}, V_{2}^{i j}$ are positive. In this case

$$
\begin{aligned}
\arg L_{\mathrm{o}}\left(\mathrm{j} \omega_{k}\right) & \\
:=\phi_{k}=\arg & G_{\mathrm{o}}\left(\mathrm{j} \omega_{k}\right) \\
+\arctan & {\left[\frac{\omega_{k}\left(\omega_{i} \tan \psi_{i}-\omega_{j} \tan \psi_{j}\right)}{\omega_{i}^{2}-\omega_{j}^{2}}\right.} \\
& \left.-\frac{\omega_{i} \omega_{j}\left(\omega_{j} \tan \psi_{i}-\omega_{i} \tan \psi_{j}\right)}{\omega_{k}\left(\omega_{i}^{2}-\omega_{j}^{2}\right)}\right]
\end{aligned}
$$

Also, $L_{\mathrm{o}}\left(\mathrm{j} \omega_{k}\right) \in R_{k} \cup S_{k}$ if and only if (iff)

$$
|\lambda| \geqslant \frac{10\left[f_{k}^{\mathrm{m}}\left(\phi_{k}\right)-\left|G_{\mathrm{o}}\left(\mathrm{j} \omega_{k}\right)\right|_{\mathrm{dB}}\right] / 20}{C_{i j}\left(\omega_{k}\right)}
$$

when $\phi_{k} \notin\left[\begin{array}{ll}\phi_{l} & \phi_{h}\end{array}\right]$

$$
|\lambda| \geqslant \frac{10\left[f_{k}\left(\phi_{k}\right)-\left|G_{\mathrm{o}}\left(\mathrm{j} \omega_{k}\right)\right|_{\mathrm{dB}}\right] / 20}{C_{i j}\left(\omega_{k}\right)}
$$

when $\phi_{k} \in\left[\begin{array}{ll}\phi_{l} & \phi_{h}\end{array}\right]$ and $f_{k}^{\mathrm{m}}\left(\phi_{k}\right) \geqslant M^{-}\left(\phi_{k}\right)$

$$
\begin{aligned}
& \frac{10\left[M^{-}\left(\phi_{k}\right)-\left|G_{\mathrm{o}}\left(\mathrm{j} \omega_{k}\right)\right|_{\mathrm{dB}} / 20\right.}{C_{i j}\left(\omega_{k}\right)} \\
& \geqslant|\lambda| \geqslant \frac{10\left[f_{k}^{\mathrm{m}}\left(\phi_{k}\right)-\left|G_{\mathrm{o}}\left(\mathrm{j} \omega_{k}\right)\right|_{\mathrm{dB}}\right] / 20}{C_{i j}\left(\omega_{k}\right)}
\end{aligned}
$$

or

$$
|\lambda| \geqslant \frac{10\left[M^{+}\left(\phi_{k}\right)-\left|G_{\mathrm{o}}\left(\mathrm{j} \omega_{k}\right)\right|_{\mathrm{dB}}\right] / 20}{C_{i j}\left(\omega_{k}\right)}
$$

when $\phi_{k} \in\left[\begin{array}{ll}\phi_{l} & \phi_{h}\end{array}\right]$ and $f_{k}^{m}\left(\phi_{k}\right)<M^{-}\left(\phi_{k}\right)$ where $C_{i j}\left(\omega_{k}\right)$ is defined in part (a).

Proof. (a) The frequency response of the PID controller is given as

$$
K(\mathrm{j} \omega)=k_{\mathrm{p}}+\mathrm{j} k_{\mathrm{d}} \omega-\mathrm{j} \frac{k_{\mathrm{i}}}{\omega}
$$

with gain and phase

$$
|K(\mathrm{j} \omega)|=\sqrt{k_{\mathrm{p}}^{2}+\left(k_{\mathrm{d}} \omega-\frac{K_{\mathrm{i}}}{\omega}\right)^{2}}
$$

and

$$
\arg K(\mathrm{j} \omega)=\arctan \left(\frac{k_{\mathrm{d}} \omega-k_{\mathrm{i}} / \omega}{k_{\mathrm{p}}}\right)
$$


respectively. Now suppose $\arg K\left(\mathrm{j} \omega_{i}\right)=\psi_{i}$ and $\arg K\left(\mathrm{j} \omega_{j}\right)=\psi_{j}$ for two frequencies $\omega_{i} \neq \omega_{j}$. Then

$$
\begin{aligned}
& k_{\mathrm{d}}-\frac{k_{\mathrm{i}}}{\omega_{i}^{2}}-\frac{k_{\mathrm{p}} \tan \left(\psi_{i}\right)}{\omega_{i}}=0 \\
& k_{\mathrm{d}}-\frac{k_{\mathrm{i}}}{\omega_{j}^{2}}-\frac{k_{\mathrm{p}} \tan \left(\psi_{j}\right)}{\omega_{j}}=0
\end{aligned}
$$

which can be written in matrix form as

$$
\left(\begin{array}{ccc}
1 & -\frac{1}{\omega_{i}^{2}} & -\frac{\tan \left(\psi_{i}\right)}{\omega_{i}} \\
1 & -\frac{1}{\omega_{j}^{2}} & -\frac{\tan \left(\psi_{j}\right)}{\omega_{j}}
\end{array}\right)\left(\begin{array}{c}
k_{\mathrm{d}} \\
k_{\mathrm{i}} \\
k_{\mathrm{p}}
\end{array}\right)=0
$$

Clearly, $\operatorname{rank}\left(\mathbf{A}_{i j}\right)=2$, since $\omega_{i} \neq \omega_{j}$ and thus the controller parameter vector is constrained to lie in the one-dimensional subspace $\operatorname{Ker}\left(\mathbf{A}_{i j}\right)$. Writing $\operatorname{Ker}\left(\mathbf{A}_{i j}\right)=$ $\lambda\left[\begin{array}{lll}V_{1}^{i j} & V_{2}^{i j} & V_{3}^{i j}\end{array}\right]^{\prime}$ gives the required expressions for $k_{\mathrm{d}}$, $k_{\mathrm{i}}$, and $k_{\mathrm{p}}$ from which the magnitude and phase expressions of $K(\mathrm{j} \omega)$ follow after some simple algebra.

(b) It is clear that when the controller gains are restricted to be non-negative, the scalars $V_{1}^{i j}$ and $V_{2}^{i j}$ must be non-negative. The conditions for $L_{\mathrm{o}}\left(\mathrm{j} \omega_{k}\right) \in$ $R_{k} \cup S_{k}$ then follow immediately from the formulation of the QFT constraints given in the previous section.

Theorem 1 shows that fixing the phase of the PID controller between $-90^{\circ}$ and $90^{\circ}$ at two distinct frequencies fixes the phase of the controller at every frequency. The Nyquist plot of the PID controller (a vertical straight line with real part $k_{\mathrm{p}}$ ) shows geometrically that in this case the three controller gains are uniquely determined (up to scaling) provided that $-90^{\circ}<\psi_{i}<\psi_{j}<90^{\circ}$ for $\omega_{i}<\omega_{j}$.

If the pure-derivative term in the controller is considered to be undesirable, the controller can be modified to the form

$$
K^{\prime}(s)=k_{\mathrm{p}}+\frac{k_{\mathrm{i}}}{s}+\frac{k_{\mathrm{d}} s}{1+s \tau}
$$

where $\tau$ is a (fixed) sufficiently small parameter. In this case, Theorem 1 can be applied with minor modifications by redefining the uncertain plant as

$$
G^{\prime}(\boldsymbol{p}, s)=\frac{G(\boldsymbol{p}, s)}{1+s \tau}
$$

and solving for the new variables $k_{\mathrm{p}}^{\prime}=k_{\mathrm{p}}+k_{\mathrm{i}} \tau$, $k_{\mathrm{i}}^{\prime}=k_{\mathrm{i}}$, and $k_{\mathrm{d}}^{\prime}=k_{\mathrm{d}}+k_{\mathrm{p}} \tau$ (see reference [15] for details).

Using essentially the same arguments a parallel result can be obtained for the $\mathrm{PDD}^{2}$ (proportional derivative-double derivative) controller $K(s)=k_{1}+$ $k_{2} s+k_{3} s^{2}$. Of course, this type of controller can be used only if the relative degree of the plant is at least two.

\section{Theorem $1^{\prime}$}

(a) Let $K(s)=k_{1}+k_{2} s+k_{3} s^{2}$ with $k_{1}, \quad k_{2}$, and $k_{3}$ real parameters. Suppose that the constraints $\arg K\left(\mathrm{j} \omega_{i}\right)=\psi_{i}$ and $\arg K\left(\mathrm{j} \omega_{j}\right)=\psi_{j}$ are imposed where $\omega_{i} \neq \omega_{j}$ and $\omega_{i} \tan \psi_{j} \leqslant \omega_{j} \tan \psi_{i}$. Then all controllers of this form are fixed up to a scaling parameter $\lambda \in \mathscr{R}$ and are parametrized as

$$
\begin{aligned}
& k_{1}=\lambda \frac{\omega_{i} \omega_{j}\left(\omega_{i} \tan \psi_{i}-\omega_{j} \tan \psi_{j}\right)}{\omega_{j} \tan \psi_{i}-\omega_{i} \tan \psi_{j}} \\
& k_{2}=\lambda \frac{\left(\omega_{i}^{2}-\omega_{j}^{2}\right) \tan \psi_{i} \tan \psi_{j}}{\omega_{j} \tan \psi_{i}-\omega_{i} \tan \psi_{j}}, \quad k_{3}=\lambda
\end{aligned}
$$

The magnitude and gain of $K(s)$ at any frequency $\omega$ is given as

$$
\begin{aligned}
& |K(\mathrm{j} \omega)| \\
& =|\lambda| \frac{\sqrt{\left[\left(\omega_{i}^{2}-\omega^{2}\right) \omega_{j} \tan \psi_{i}+\left(\omega^{2}-\omega_{j}^{2}\right) \omega_{i} \tan \psi_{j}\right]^{2}}}{+\omega^{2}\left(\omega_{i}^{2}-\omega_{j}^{2}\right) \tan ^{2} \psi_{i} \tan ^{2} \psi_{j}} \\
& :=|\lambda| C_{i j}(\omega)
\end{aligned}
$$

and

$\arg K(\mathrm{j} \omega)$

$$
=\arctan \left[\frac{\omega\left(\omega_{i}^{2}-\omega_{j}^{2}\right) \tan \psi_{i} \tan \psi_{j}}{\left(\omega_{i}^{2}-\omega^{2}\right) \omega_{j} \tan \psi_{i}+\left(\omega^{2}-\omega_{j}^{2}\right) \omega_{i} \tan \psi_{j}}\right]
$$

respectively.

(b) If the controller gains $k_{1}, k_{2}$, and $k_{3}$ are restricted to be non-negative, then the constraints $\arg K\left(\mathrm{j} \omega_{i}\right)=$ $\psi_{i}$ and $\arg K\left(\mathrm{j} \omega_{j}\right)=\psi_{j}$ with $0<\psi_{i}<\pi$ and $0<\psi_{j}<\pi$ are feasible if and only if (iff)

$$
\frac{\omega_{i} \tan \psi_{i}-\omega_{j} \tan \psi_{j}}{\omega_{j} \tan \psi_{i}-\omega_{i} \tan \psi_{j}} \geqslant 0
$$

and

$$
\frac{\left(\omega_{i}-\omega_{j}\right) \tan \psi_{i} \tan \psi_{j}}{\omega_{j} \tan \psi_{i}-\omega_{i} \tan \psi_{j}} \geqslant 0
$$

In this case

$$
\begin{aligned}
\arg & L_{\mathrm{o}}\left(\mathrm{j} \omega_{k}\right) \\
:= & \phi_{k}=\arg G_{\mathrm{o}}\left(\mathrm{j} \omega_{k}\right) \\
& +\arctan \left[\frac{\omega_{k}\left(\omega_{i}^{2}-\omega_{j}^{2}\right) \tan \psi_{i} \tan \psi_{j}}{\left(\omega_{i}^{2}-\omega_{k}^{2}\right) \omega_{j} \tan \psi_{i}+\left(\omega_{k}^{2}-\omega_{j}^{2}\right) \omega_{i} \tan \psi_{j}}\right]
\end{aligned}
$$


Also, $L_{\mathrm{o}}\left(\mathrm{j} \omega_{k}\right) \in R_{k} \cup S_{k}$ iff

$$
|\lambda| \geqslant \frac{10\left[f_{k}^{\mathrm{m}}\left(\phi_{k}\right)-\left|G_{\mathrm{o}}\left(\mathrm{j} \omega_{k}\right)\right|_{\mathrm{dB}}\right] / 20}{C_{i j}\left(\omega_{k}\right)}
$$

when $\phi_{k} \notin\left[\begin{array}{ll}\phi_{l} & \phi_{h}\end{array}\right]$

$$
|\lambda| \geqslant \frac{10\left[f_{k}\left(\phi_{k}\right)-\left|G_{\mathrm{o}}\left(\mathrm{j} \omega_{k}\right)\right|_{\mathrm{dB}}\right] / 20}{C_{i j}\left(\omega_{k}\right)}
$$

when $\phi_{k} \in\left[\begin{array}{ll}\phi_{l} & \phi_{h}\end{array}\right]$ and $f_{k}^{\mathrm{m}}\left(\phi_{k}\right) \geqslant M^{-}\left(\phi_{k}\right)$

$$
\begin{aligned}
& \frac{10\left[M^{-}\left(\phi_{k}\right)-\left|G_{\mathrm{o}}\left(\mathrm{j} \omega_{k}\right)\right|_{\mathrm{dB}}\right] / 20}{C_{i j}\left(\omega_{k}\right)} \\
& \geqslant|\lambda| \geqslant \frac{10\left[f_{k}^{\mathrm{m}}\left(\phi_{k}\right)-\left|G_{\mathrm{o}}\left(\mathrm{j} \omega_{k}\right)\right|_{\mathrm{dB}}\right] / 20}{C_{i j}\left(\omega_{k}\right)}
\end{aligned}
$$

or

$$
|\lambda| \geqslant \frac{10\left[M^{+}\left(\phi_{k}\right)-\left|G_{\mathrm{o}}\left(\mathrm{j} \omega_{k}\right)\right|_{\mathrm{dB}}\right] / 20}{C_{i j}\left(\omega_{k}\right)}
$$

when $\phi_{k} \in\left[\begin{array}{ll}\phi_{l} & \phi_{h}\end{array}\right]$ and $f_{k}^{\mathrm{m}}\left(\phi_{k}\right)<M^{-}\left(\phi_{k}\right)$ where $C_{i j}\left(\omega_{k}\right)$ is defined in part (a).

Proof. This follows in a similar way to the proof of Theorem 1. Similar conclusions can also be drawn about the gain and phase of the open-loop system and its permissible regions subject to QFT constraints (details are omitted).

\subsection{Phase-lead/lag controller}

Next the case of a first-order phase-lead (phaseadvance) controller is considered. The dual result for a phase-lag controller also follows easily.

\section{Theorem 2}

Let $K(s)=k(s+b) /(s+a)$ with $a>b>0$ ('phase-lead' controller). Then the constraints $\arg K\left(\mathrm{j} \omega_{i}\right)=\psi_{i}$ and $\arg K\left(\mathrm{j} \omega_{j}\right)=\psi_{j}$ for two distinct frequencies $\omega_{i} \neq \omega_{j}$ with $0<\psi_{i}<90^{\circ}$ and $0<\psi_{j}<90^{\circ}$ are feasible if and only if the following two conditions are satisfied

$$
\lambda:=\frac{\left(\omega_{i}^{2}-\omega_{j}^{2}\right) \tan \psi_{i} \tan \psi_{j}}{\omega_{i} \tan \psi_{j}-\omega_{j} \tan \psi_{i}}>0
$$

and

$$
c:=\frac{\omega_{i} \omega_{j}\left(\omega_{j} \tan \psi_{j}-\omega_{i} \tan \psi_{i}\right)}{\omega_{i} \tan \psi_{j}-\omega_{j} \tan \psi_{i}}<0
$$

In this case, the quadratic equation

$$
b^{2}+\lambda b+c=0
$$

has one positive root $b_{+}$and one negative root $b_{-}$ and the controller parameters and are determined uniquely as $b=b_{+}$and $a=b_{+}+\lambda=-b_{-}$. In addition

$$
\begin{aligned}
\arg L_{\mathrm{o}}\left(\mathrm{j} \omega_{k}\right):= & \phi_{k}=\arg G_{\mathrm{o}}\left(\mathrm{j} \omega_{k}\right)+\arctan \left(\frac{\omega_{k}}{b}\right) \\
& -\arctan \left(\frac{\omega_{k}}{a}\right)
\end{aligned}
$$

and $L_{\mathrm{o}}\left(\mathrm{j} \omega_{k}\right) \in R_{k} \cup S_{k}$ iff

$$
k \geqslant \frac{10\left[f_{k}^{\mathrm{m}}\left(\phi_{k}\right)-\left|G_{\mathrm{o}}\left(\mathrm{j} \omega_{k}\right)\right|_{\mathrm{dB}}\right] / 20}{C\left(\omega_{k}\right)}
$$

when $\phi_{k} \notin\left[\begin{array}{ll}\phi_{l} & \phi_{h}\end{array}\right]$

$$
k \geqslant \frac{10\left[f_{k}\left(\phi_{k}\right)-\left|G_{\mathrm{o}}\left(\mathrm{j} \omega_{k}\right)\right|_{\mathrm{dB}}\right] / 20}{C\left(\omega_{k}\right)}
$$

when $\phi_{k} \in\left[\begin{array}{ll}\phi_{l} & \phi_{h}\end{array}\right]$ and $f_{k}^{\mathrm{m}}\left(\phi_{k}\right) \geqslant M^{-}\left(\phi_{k}\right)$

$$
\begin{aligned}
& \frac{10\left[M^{-}\left(\phi_{k}\right)-\left|G_{\mathrm{o}}\left(\mathrm{j} \omega_{k}\right)\right|_{\mathrm{dB}}\right] / 20}{C\left(\omega_{k}\right)} \\
& \geqslant k \geqslant \frac{10\left[f_{k}^{\mathrm{m}}\left(\phi_{k}\right)-\left|G_{\mathrm{o}}\left(\mathrm{j} \omega_{k}\right)\right|_{\mathrm{dB}}\right] / 20}{C\left(\omega_{k}\right)}
\end{aligned}
$$

or

$$
k \geqslant \frac{10\left[M^{+}\left(\phi_{k}\right)-\left|G_{\mathrm{o}}\left(\mathrm{j} \omega_{k}\right)\right|_{\mathrm{dB}}\right] / 20}{C\left(\omega_{k}\right)}
$$

when $\phi_{k} \in\left[\begin{array}{ll}\phi_{l} & \phi_{h}\end{array}\right]$ and $f_{k}^{\mathrm{m}}\left(\phi_{k}\right)<M^{-}\left(\phi_{k}\right)$ where $C(\omega)=\sqrt{\left(b^{2}+\omega^{2}\right) /\left(a^{2}+\omega^{2}\right)}$.

Proof. The frequency response of the phase-lead controller is given as

$$
K(\mathrm{j} \omega)=k \frac{\mathrm{j} \omega+b}{\mathrm{j} \omega+a}
$$

with the gain and phase

$$
|K(\mathrm{j} \omega)|=k \sqrt{\frac{\omega^{2}+b^{2}}{\omega^{2}+a^{2}}}
$$

and

$$
\arg K(\mathrm{j} \omega)=\arctan \left(\frac{\omega}{b}\right)-\arctan \left(\frac{\omega}{a}\right)
$$

respectively. Now suppose $\arg K\left(\mathrm{j} \omega_{i}\right)=\psi_{i}$ and $\arg K\left(j \omega_{j}\right)=\psi_{j}$ are fixed for two frequencies $\omega_{i} \neq \omega_{j}$. Then

$$
\begin{aligned}
& \arctan \left(\frac{\omega_{i}}{b}\right)-\arctan \left(\frac{\omega_{i}}{a}\right)=\psi_{i} \\
& \arctan \left(\frac{\omega_{j}}{b}\right)-\arctan \left(\frac{\omega_{j}}{a}\right)=\psi_{j}
\end{aligned}
$$

Using the trigonometric identity

$$
\tan (\alpha-\beta)=\frac{\tan \alpha-\tan \beta}{1+\tan \alpha \tan \beta}
$$


gives (after some algebra)

$$
\begin{aligned}
& \omega_{i}^{2} \tan \psi_{i}-\omega_{i} a+\omega_{i} b+a b \tan \psi_{i}=0 \\
& \omega_{j}^{2} \tan \psi_{j}-\omega_{j} a+\omega_{j} b+a b \tan \psi_{j}=0
\end{aligned}
$$

Multiplying the first equation by $\tan \psi_{j}$, the second equation by $\tan \psi_{i}$, and subtracting the resulting two equations gives

$$
a=b+\frac{\left(\omega_{i}^{2}-\omega_{j}^{2}\right) \tan \psi_{i} \tan \psi_{j}}{\omega_{i} \tan \psi_{j}-\omega_{j} \tan \psi_{i}}:=b+\lambda
$$

Since $a>b$ for a phase-lead controller, then $\lambda>0$. Substituting for $a=b+\lambda$ in equation (54) leads to the quadratic equation

$$
\begin{aligned}
b^{2}+ & \frac{\left(\omega_{i}^{2}-\omega_{j}^{2}\right) \tan \psi_{i} \tan \psi_{j}}{\omega_{i} \tan \psi_{j}-\omega_{j} \tan \psi_{i}} b \\
+ & \frac{\omega_{i} \omega_{j}\left(\omega_{j} \tan \psi_{j}-\omega_{i} \tan \psi_{i}\right)}{\omega_{i} \tan \psi_{j}-\omega_{j} \tan \psi_{i}}=0
\end{aligned}
$$

This must have a positive root $b_{+}$if the constraints are feasible, so that $a=b_{+}+\lambda>b=b>0$. To see that at most one of the two roots of the quadratic equation

$$
b=\frac{-\lambda \pm \sqrt{\lambda^{2}-4 c}}{2}
$$

is positive, note that the transfer functions $(s+b) /$ $(s+a)$ and $(s-a) /(s-b)$ have identical phase responses; hence, if one root of the quadratic is $b$, the other root must be $-a$. Formally, when $\lambda>0$ the roots of the quadratic can be classified as follows:

$c<0$ : One positive $\left(b_{+}\right)$and one negative $(b)$ root $c=0$ : zero and negative $(b=-\lambda)$ roots

$0<c \leqslant \lambda^{2} / 4$ : here $\sqrt{\lambda^{2}-4 c}<\lambda$ so both roots are negative

$c>\lambda^{2} / 4$ : complex conjugate roots

Therefore parameters $a$ and $b$ with $a>b>0$ are uniquely determined from the two phase conditions when $\lambda>0$ and $c<0$. To show that $b_{-}=-a$ note that

$$
\begin{aligned}
a & =b_{+}+\lambda=\frac{-\lambda+\sqrt{\lambda^{2}-4 c}}{2}+\lambda \\
& =\frac{\lambda+\sqrt{\lambda^{2}-4 c}}{2}=-b_{-}
\end{aligned}
$$

The phase equation for $L_{\mathrm{o}}\left(\mathrm{j} \omega_{k}\right)$ is immediate, while the gain inequalities on $k$ for $L_{\mathrm{o}}\left(\mathrm{j} \omega_{k}\right) \in R_{k} \cup S_{k}$ follow directly from the discussion of the previous section after noting that $\left|K\left(\mathrm{j} \omega_{k}\right)\right|=k C\left(\omega_{k}\right)$.

\section{Example 1}

Consider the following cases.

1. $\omega_{i}=1 \mathrm{rad} / \mathrm{s}, \omega_{j}=4 \mathrm{rad} / \mathrm{s}, \psi_{i}=10^{\circ}$, and $\psi_{j}=30^{\circ}$. This gives $\lambda=11.9339, c=-66.6806$, and so the constraints are feasible. The quadratic equation gives $b=b_{+}=4.1467$ and $a=b_{+}+\lambda=-b_{-}=16.0806$.

2. $\omega_{i}=1 \mathrm{rad} / \mathrm{s}, \omega_{j}=4 \mathrm{rad} / \mathrm{s}, \psi_{i}=60^{\circ}$, and $\psi_{j}=10^{\circ}$. This gives $\lambda=0.6785, c=0.6083$, and the roots of the quadratic are complex: $b_{1,2}=-0.3393 \pm \mathrm{j} 0.7023$. The constraints are infeasible.

3. $\omega_{i}=1 \mathrm{rad} / \mathrm{s}, \omega_{j}=4 \mathrm{rad} / \mathrm{s}, \psi_{i}=-10^{\circ}$, and $\psi_{j}=30^{\circ}$. Clearly the constraints are infeasible for a lead (or a lag) controller. This gives $\lambda=1.1905$, $c=7.7518$, and the quadratic has complex roots $b_{1,2}=-0.5953 \pm \mathrm{j} 2.7198$.

4. $\omega_{i}=1 \mathrm{rad} / \mathrm{s}, \omega_{j}=4 \mathrm{rad} / \mathrm{s}, \psi_{i}=-10^{\circ}$ and $\psi_{j}=-30^{\circ}$. Clearly the constraints are infeasible for a phaselead controller (but not for a phase-lag controller). This gives $\lambda=-11.9339, \quad c=-66.6806$, while the quadratic equation gives $b_{+}=16.0806$ and $b_{-}=-4.1467$.

The corresponding result for a phase-lag controller is as follows.

\section{Theorem $2^{\prime}$}

Let $K(s)=k(s+b) /(s+a)$ with $b>a>0$ ('phase-lag' controller). Then the constraints $\arg K\left(\mathrm{j} \omega_{i}\right)=\psi_{i}$ and $\arg K\left(\mathrm{j} \omega_{j}\right)=\psi_{j}$ for two distinct frequencies $\omega_{i} \neq \omega_{j}$ with $-90^{\circ}<\psi_{i}<0^{\circ}$ and $-90^{\circ}<\psi_{j}<0^{\circ}$ are feasible if and only if the following two conditions are satisfied

$$
\lambda:=\frac{\left(\omega_{i}^{2}-\omega_{j}^{2}\right) \tan \psi_{i} \tan \psi_{j}}{\omega_{i} \tan \psi_{j}-\omega_{j} \tan \psi_{i}}<0
$$

and

$$
c:=\frac{\omega_{i} \omega_{j}\left(\omega_{j} \tan \psi_{j}-\omega_{i} \tan \psi_{i}\right)}{\omega_{i} \tan \psi_{j}-\omega_{j} \tan \psi_{i}}<0
$$

In this case, the quadratic equation

$$
b^{2}+\lambda b+c=0
$$

has one positive root $b_{+}$and one negative root $b_{-}$and the controller parameters $b$ and $a$ are determined uniquely as $b=b_{+}$and $a=b_{+}+\lambda=-b_{-}$. In addition

$$
\begin{aligned}
\arg L_{\mathrm{o}}\left(\mathrm{j} \omega_{k}\right):= & \phi_{k}=\arg G_{\mathrm{o}}\left(\mathrm{j} \omega_{k}\right)+\arctan \left(\frac{\omega_{k}}{b}\right) \\
& -\arctan \left(\frac{\omega_{k}}{a}\right)
\end{aligned}
$$

and $L_{\mathrm{o}}\left(\mathrm{j} \omega_{k}\right) \in R_{k} \cup S_{j k}$ iff

$$
k \geqslant \frac{10\left[f_{k}^{\mathrm{m}}\left(\phi_{k}\right)-\left|G_{\mathrm{o}}\left(\mathrm{j} \omega_{k}\right)\right|_{\mathrm{dB}}\right] / 20}{C\left(\omega_{k}\right)}
$$


when $\phi_{k} \notin\left[\begin{array}{ll}\phi_{l} & \phi_{h}\end{array}\right]$

$$
k \geqslant \frac{10\left[f_{k}\left(\phi_{k}\right)-\left|G_{\mathrm{o}}\left(\mathrm{j} \omega_{k}\right)\right|_{\mathrm{dB}}\right] / 20}{C\left(\omega_{k}\right)}
$$

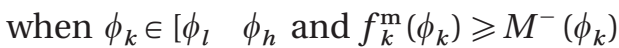

$$
\begin{aligned}
& \frac{10\left[M^{-}\left(\phi_{k}\right)-\left|G_{\mathrm{o}}\left(\mathrm{j} \omega_{k}\right)\right|_{\mathrm{dB}}\right] / 20}{C\left(\omega_{k}\right)} \\
& \geqslant k \geqslant \frac{10\left[f_{k}^{\mathrm{m}}\left(\phi_{k}\right)-\left|G_{\mathrm{o}}\left(\mathrm{j} \omega_{k}\right)\right|_{\mathrm{dB}}\right] / 20}{C\left(\omega_{k}\right)}
\end{aligned}
$$

or

$$
k \geqslant \frac{10\left[M^{+}\left(\phi_{k}\right)-\left|G_{\mathrm{o}}\left(\mathrm{j} \omega_{k}\right)\right|_{\mathrm{dB}}\right] / 20}{C\left(\omega_{k}\right)}
$$

when $\phi_{k} \in\left[\begin{array}{ll}\phi_{l} & \phi_{h}\end{array}\right]$ and $f_{k}^{\mathrm{m}}\left(\phi_{k}\right)<M^{-}\left(\phi_{k}\right)$ where $C(\omega)=\sqrt{\left(b^{2}+\omega^{2}\right) /\left(a^{2}+\omega^{2}\right) .}$

Proof. This follows along similar lines to the proof of Theorem 2.

Theorems 2 and $2^{\prime}$ show that fixing the phases of the phase-lead or phase-lag controller in the intervals $\left(0^{\circ}, 90^{\circ}\right)$ or $\left(-90^{\circ}, 0^{\circ}\right)$ respectively determines uniquely the dynamic part of the controller when the the constraints are feasible. Feasibility of the constraints is easily checked from two sign conditions, and the controller parameters are determined by solving a quadratic equation.

\subsection{Second-order controller with complex poles or zeros}

Finally the case is considered of a second-order controller with complex (conjugate) poles. The dual result of a second-order controller with complex zeros then follows immediately.

\section{Theorem 3}

(a) Let $K(s)=k /\left(s^{2}+2 \zeta \omega_{n} s+\omega_{n}^{2}\right)$ with $\omega_{n}>0$ and $0<\zeta<1$ ('complex-pole second-order lag'). Then the constraints $\arg K\left(\mathrm{j} \omega_{i}\right)=\psi_{i}$ and $\arg K\left(\mathrm{j} \omega_{j}\right)=\psi_{j}$ for two distinct frequencies $\omega_{i} \neq \omega_{j}$ with $-180^{\circ} \mathrm{M} \psi_{i}<0^{\circ}$ and $-180^{\circ}<\psi_{j}<0^{\circ}$ and $\psi_{i} \neq \psi_{j}$ are feasible if and only if the following conditions are satisfied:

1. If $\psi_{i} \neq-90^{\circ}$ and $\psi_{j} \neq-90^{\circ}$

$$
\lambda=\frac{\omega_{j} \tan \psi_{j}-\omega_{i} \tan \psi_{i}}{\omega_{i} \tan \psi_{j}-\omega_{j} \tan \psi_{i}}>0
$$

and

$$
0<\zeta:=\frac{\tan \psi_{i}}{2}\left(\sqrt{\frac{\omega_{j} \lambda}{\omega_{i}}}-\sqrt{\frac{\omega_{i}}{\lambda \omega_{j}}}\right)<1
$$

in which case $\omega_{n}$ and $\zeta$ are defined uniquely as $\omega_{n}=\sqrt{\omega_{i} \omega_{j} \lambda}$ and via equation (62) respectively.

2. If $\psi_{j}=-90^{\circ}$ then either of the following two conditions must hold: $\psi_{i} \in\left(-90^{\circ}, 0^{\circ}\right)$ and $\omega_{j}<\omega_{i}$ or $\psi_{i} \in\left(-180^{\circ},-90^{\circ}\right)$ and $\omega_{j}>\omega_{i}$, in addition to the condition

$$
\zeta:=\frac{\left(\omega_{j}^{2}-\omega_{i}^{2}\right) \tan \psi_{i}}{2 \omega_{i} \omega_{j}}<1
$$

in which case $\omega_{n}$ and $\zeta$ are uniquely determined as $\omega_{n}=\omega_{j}$ and via equation (63) respectively.

3. If $\psi_{i}=-90^{\circ}$ then either of the following two conditions must hold: $\psi_{j} \in\left(-90^{\circ}, 0^{\circ}\right)$ and $\omega_{i}<\omega_{j}$ or $\psi_{j} \in\left(-180^{\circ},-90^{\circ}\right)$ and $\omega_{i}>\omega_{j}$, in addition to the condition

$$
\zeta:=\frac{\left(\omega_{i}^{2}-\omega_{j}^{2}\right) \tan \psi_{j}}{2 \omega_{i} \omega_{j}}<1
$$

in which case $\omega_{n}$ and $\zeta$ are uniquely determined as $\omega_{n}=\omega_{i}$ and via equation (64) respectively.

(b) When the phase conditions are feasible then

$$
\arg L_{\mathrm{o}}\left(\mathrm{j} \omega_{k}\right):=\phi_{k}=\arg G_{\mathrm{o}}\left(\mathrm{j} \omega_{k}\right)+\arctan \left(\frac{2 \zeta \omega_{n} \omega_{k}}{\omega_{n}^{2}-\omega_{k}^{2}}\right)
$$

and $L_{\mathrm{o}}\left(\mathrm{j} \omega_{k}\right) \in R_{k} \cup S_{k}$ iff

$$
k \geqslant \frac{10\left[f_{k}^{\mathrm{m}}\left(\phi_{k}\right)-\left|G_{\mathrm{o}}\left(\mathrm{j} \omega_{k}\right)\right|_{\mathrm{dB}}\right] / 20}{C\left(\omega_{k}\right)}
$$

when $\phi_{k} \notin\left[\begin{array}{ll}\phi_{l} & \phi_{h}\end{array}\right]$

$$
k \geqslant \frac{10\left[f_{k}\left(\phi_{k}\right)-\left|G_{\mathrm{o}}\left(\mathrm{j} \omega_{k}\right)\right|_{\mathrm{dB}}\right] / 20}{C\left(\omega_{k}\right)}
$$

when $\phi_{k} \in\left[\begin{array}{ll}\phi_{l} & \phi_{h}\end{array}\right]$ and $f_{k}^{\mathrm{m}}\left(\phi_{k}\right) \geqslant M^{-}\left(\phi_{k}\right)$

$$
\begin{aligned}
& \frac{10\left[M^{-}\left(\phi_{k}\right)-\left|G_{\mathrm{o}}\left(\mathrm{j} \omega_{k}\right)\right|_{\mathrm{dB}}\right] / 20}{C\left(\omega_{k}\right)} \\
& \geqslant k \geqslant \frac{10\left[f_{k}^{\mathrm{m}}\left(\phi_{k}\right)-\left|G_{\mathrm{o}}\left(\mathrm{j} \omega_{k}\right)\right|_{\mathrm{dB}}\right] / 20}{C\left(\omega_{k}\right)}
\end{aligned}
$$

or

$$
k \geqslant \frac{10\left[M^{+}\left(\phi_{k}\right)-\left|G_{\mathrm{o}}\left(\mathrm{j} \omega_{k}\right)\right|_{\mathrm{dB}}\right] / 20}{C\left(\omega_{k}\right)}
$$

when

$$
\phi_{k} \in\left[\begin{array}{ll}
\phi_{l} & \phi_{h}
\end{array}\right] \quad \text { and } \quad f_{k}^{\mathrm{m}}\left(\phi_{k}\right)<M^{-}\left(\phi_{k}\right)
$$

where $C(\omega)=1 / \sqrt{\left(\omega_{n}-\omega\right)^{2}+4 \zeta^{2} \omega_{n}^{2} \omega^{2}}$.

Proof. The frequency response of the controller is given by

$$
K(\mathrm{j} \omega)=\frac{k}{\omega_{n}^{2}-\omega^{2}+2 \mathrm{j} \xi \omega_{n} \omega}
$$


from which its magnitude and phase responses can be obtained as

$$
|K(\mathrm{j} \omega)|=\frac{k}{\sqrt{\left(\omega_{n}^{2}-\omega^{2}\right)^{2}+4 \xi^{2} \omega_{n}^{2} \omega^{2}}}=k C(\omega)
$$

and

$$
\arg K(\mathrm{j} \omega)=\arctan \left(\frac{2 \zeta \omega_{n} \omega}{\omega_{n}^{2}-\omega^{2}}\right)
$$

respectively. Setting $\arg K\left(\mathrm{j} \omega_{i}\right)=\psi_{i}$ and $\arg K\left(\mathrm{j} \omega_{j}\right)=\psi_{j}$ gives

$$
\frac{2 \zeta \omega_{n} \omega_{i}}{\omega_{n}^{2}-\omega_{i}^{2}}=\tan \psi_{i} \quad \text { and } \quad \frac{2 \zeta \omega_{n} \omega_{j}}{\omega_{n}^{2}-\omega_{j}^{2}}=\tan \psi_{j}
$$

for $\psi_{i} \neq-90^{\circ}$ and $\psi_{j} \neq-90^{\circ}$. Solving simultaneously the above two equations gives

$$
\omega_{n}^{2}=\frac{\omega_{i} \omega_{j}\left(\omega_{j} \tan \psi_{j}-\omega_{i} \tan \psi_{i}\right)}{\omega_{i} \tan \psi_{j}-\omega_{j} \tan \psi_{i}}
$$

which defines $\omega_{n}$ uniquely iff $\lambda>0$. Substituting into equation (62) then gives the expression for $\zeta$ and the corresponding condition for an underdamped response $(0<\zeta<1)$. When $\psi_{j}=-90^{\circ}$ then $\omega_{n}=\omega_{j}$ and hence $\zeta$ is given by equation (63). This is positive when $\left(\omega_{j}-\omega_{i}\right) \tan \psi_{i}>0$, from which the two stated conditions follow. Finally, the phase equation for $L_{\mathrm{o}}\left(\mathrm{j} \omega_{k}\right)$ follows immediately, while the gain conditions for $\left.L_{\mathrm{o}} \mathrm{j} \omega_{k}\right) \in R_{k} \cup S_{k}$ can be derived from the discussion in the previous section on QFT constraints.

Again, Theorem 3 shows that fixing the phase of the controller at two distinct frequencies determines completely the dynamic part of the controller when the constraints are feasible. In the theorem formulation the controller is restricted to be underdamped. This restriction can be removed, if required, by ignoring throughout the $\zeta<1$ condition. An almost identical procedure may be used to determine the dynamic part of a minimum-phase non-proper controller $k\left(s^{2}+2 \zeta \omega_{n} s+\omega_{n}^{2}\right)$ from its two phases in the interval $\left(0^{\circ}, 180^{\circ}\right)$ at two distinct frequencies $\omega_{i}$ and $\omega_{j}$; details are omitted.

In all three cases considered above simple gain conditions on the nominal open-loop gain have been derived, so that the QFT robust stability and performance constraints are satisfied. These are of the form $L_{\mathrm{o}}\left(\mathrm{j} \omega_{k}\right) \in R_{k} \cup S_{k}$, which for a fixed phase $\arg L_{\mathrm{o}}\left(\mathrm{j} \omega_{k}\right)=\phi_{k}$ correspond to gain intervals

$$
\left[k_{1}\left(\omega_{i}, \phi_{k}\right), k_{2}\left(\omega_{i}, \phi_{k}\right)\right] \cup\left[k_{3}\left(\omega_{i}, \phi_{k}\right), \infty\right]
$$

where $i=1,2, \ldots, N$. Thus the optimization problem takes the form: 'Minimize the optimization criterion (i.e. crossover frequency, closed-loop bandwidth, asymptotic open-loop gain, etc.), so that for each design frequency $\omega_{i}, i=1,2, \ldots, N, L_{\mathrm{o}}\left(\mathrm{j} \omega_{k}\right) \in S_{k} \cup R_{k}$ and Nyquist's encirclement criterion is satisfied'. Since for the three types of controllers described above the phase of the nominal open-loop system is completely determined once two controller phases have been fixed, the following algorithm can be used for solving the optimization problem.

\subsection{Optimization algorithm}

1. Obtain a phase array $\Phi$ by discretizing the phase interval $\left(-360^{\circ}, 0^{\circ}\right]$.

2. Select any two distinct frequencies $\omega_{i}$ and $\omega_{j}$ in the set of design frequencies $\left(\omega_{1}, \omega_{2}, \ldots, \omega_{N}\right)$.

3. Calculate the phase intervals $\Phi_{k} \subseteq \Phi$ and $\Phi_{l} \subseteq \Phi$ in which the nominal open-loop phase $\arg L_{\mathrm{o}}(\mathrm{j} \omega)$ can vary at $\omega=\omega_{k}$ and $\omega=\omega_{l}$ respectively. These depend on the type of controller to be designed, e.g. for a PID controller they lie within $\pm 90^{\circ}$ of $\arg G\left(\boldsymbol{p}_{\mathrm{o}}, \omega_{k}\right)$ and $\arg G\left(\boldsymbol{p}_{\mathrm{o}}, \omega_{l}\right)$, etc.

4. Initialize an $m \times n$ array $F$ where $m$ and $n$ are the sizes of $\Phi_{k}$ and $\Phi_{l}$ respectively, to contain the value of the objective function (crossover frequency, closed-loop bandwidth, asymptotic gain, etc.) for each phase pair. Also, initialize $m \times n$ controller parameter arrays to contain the parameters, e.g. $\left(k_{\mathrm{p}}, k_{\mathrm{d}}, k_{\mathrm{i}}\right)$ for a PID controller, $\left(k_{\mathrm{o}}, a, b\right)$ for a phase-lead/lag controller, or $\left(k_{\mathrm{o}}, \omega_{n}, \zeta\right)$ for a second-order controller with complex poles/zeros.

5. For each $\left(\Phi_{k}(i), \Phi_{l}(j)\right) \in \Phi_{k} \times \Phi_{l} \subseteq \mathscr{R}^{m \times n}$ :

(a) Calculate $\psi_{i}=\Phi_{k}(i)-\arg G\left(\boldsymbol{p}_{0}, \omega_{k}\right)$ and $\psi_{j}=$ $\Phi_{l}(j)-\arg G\left(\boldsymbol{p}_{\mathrm{o}}, \omega_{l}\right)$.

(b) Determine a controller $K(s)$ of one of the above types, such that $\arg K\left(\mathrm{j} \omega_{k}\right)=\psi_{i}$ and $\arg K\left(\mathrm{j} \omega_{l}\right)=\psi_{j}$. If these phase constraints are infeasible, set $F(i, j)=\infty$ and consider the next phase pair $\left(\Phi_{k}(i), \Phi_{l}(j)\right)$.

(c) Find the minimum value of gain $k_{\mathrm{o}}>0$ such that $L_{\mathrm{o}}\left(\mathrm{j} \omega_{q}\right)=k_{\mathrm{o}} K\left(\mathrm{j} \omega_{q}\right) G\left(\boldsymbol{p}_{\mathrm{o}}, \mathrm{j} \omega_{q}\right) \in R_{q} \cup S_{q}$ for all $q=1,2, \ldots, N$ and $L_{\mathrm{o}}(\mathrm{j} \omega)$ satisfies Nyquist's encirclement criterion. If no such gain $k_{\mathrm{o}}$ exists, set $F(i, j)=\infty$ and consider the next phase pair $\left(\Phi_{k}(i), \Phi_{l}(j)\right)$.

(d) Calculate the value of the objective function (crossover frequency, closed-loop bandwidth, asymptotic gain, etc.) corresponding to the designed $L_{\mathrm{o}}(\mathrm{j} \omega)$ and assign it to the $(i, j)$ th element of $F$. Save also the controller parameters to the corresponding entries of the parameter arrays.

6. At the end of all $m \times n$ iterations, calculate $\gamma_{\mathrm{o}}=$ $\min _{(i, j) \in \Phi_{k} \times \Phi_{l}}(F)$ and $\left(i^{*}, j^{*}\right) \in \operatorname{argmin}(F)$. If $\gamma_{\mathrm{o}}=\infty$ 
the QFT constraints are infeasible; otherwise the optimal cost is $\gamma_{\mathrm{o}}$ and the optimal controller parameters can be obtained from the $\left(i^{*}, j^{*}\right)$ th elements of the controller-parameter arrays.

A few remarks can be made on the algorithm.

1. In step 1 of the algorithm the phase discretization of the interval $\left(-360^{\circ}, 0^{\circ}\right]$ results in a phase grid $\Phi$, typically equally spaced. In practice, $50-100$ phases are adequate. It is helpful to calculate the performance bounds ('Horowitz tracking', 'Horowitz disturbance-rejection' templates and ' $U$-contour) over the same phase grid.

2. In principle any two frequencies $\omega_{k}$ and $\omega_{l}$ can be selected from the set of design frequencies in step 2. In general, selecting these frequencies reasonably far apart (for minimum numerical sensitivity) works well in practice. A common-sense rule is to choose frequencies at which the controller can introduce a wide range of phase without conflicting with the QFT constraints or the expected characteristics of the system; for example, if the nominal plant is of type zero and the controller introduces integral action, the open-loop phase at very low frequencies will be near -90 degrees, and therefore frequencies in this range should not be selected.

3. In steps 3 and 5(a) of the algorithm all phase calculations can be performed modulo $-360^{\circ}$. This restricts the phase interval of interest to the range $\left(-360^{\circ}, 0^{\circ}\right.$.

4. Since the phase of $L_{\mathrm{o}}(\mathrm{j} \omega)$ is completely determined when two controller phases are fixed, the calculation of the minimum gain in step 5(c) is straightforward. For example, one possible method is to calculate the minimum distance between the plant and the corresponding 'open' performance bounds and check whether this amount of gain brings the high design frequencies within the $\mathscr{U}$-contour, together with a stability test. Checking the total number of encirclements required for stability is also straightforward and can be performed by purely graphical means (i.e. by counting the crossings of the $-180^{\circ}$ line and their directions). See references [8] and [9] for details. Note also that a frequency grid 'denser' than the set of design frequencies must typically be used for this purpose.

5. Step 5(c) requires the calculations of the performance bounds at arbitrary phases, which may not coincide with the discretized phases of array $\Phi$. There is no difficulty, however, in estimating the performance gains from adjacent phase points, e.g. using linear interpolation. Alternatively, the performance bounds may be calculated exactly at these phases to arbitrary accuracy using a bisection algorithm implemented between steps 5(b) and 5(c). In practice, however, it is sufficient to substitute each point with the one that is closest on the predefined phase grid.

\section{DESIGN EXAMPLE}

In this example some of the techniques described above are applied for designing a robust force controller for a non-linear hydraulic actuator interacting with an uncertain environment. The linearized model of the actuator is based on references [25] and [26] from where full modelling details can be found. A schematic of the hydraulic actuator is shown in Fig. 5. Uncertainty in the model arises from variations in operating-point dependent parameters, changes in the environment, and changes in the hydraulic actuator's functions.

\subsection{Controller design}

The overall transfer function between the measured contact force $F(s)$ and applied control voltage $V(s)$ is given as

$$
\begin{aligned}
G(s)= & \frac{F(s)}{V(s)}=\frac{k_{\mathrm{sp}}}{\tau s+1} \\
& \times\left[\frac{K_{s} k_{e}\left(A_{\mathrm{i}}+A_{\mathrm{o}}\right)}{\left(K_{p}+C s\right)\left(m_{a} s^{2}+d s+k_{e}\right)+\left(A_{\mathrm{i}}^{2}+A_{\mathrm{o}}^{2}\right) s}\right]
\end{aligned}
$$

Here $k_{s}$ and $d_{s}$ represent sensor stiffness and damping respectively. The sensor connects the actuator's piston of mass $m_{a}$ to the environment, represented by a mass $m_{e}$, stiffness $k_{e}$, and damping $d_{e}$. Further, $A_{\mathrm{i}}$ and $A_{\mathrm{o}}$ represent the effective inner and outer areas of the piston, $\tau$ and $k_{\mathrm{sp}}$ are gains describing the valve dynamics, while $K_{s}$ and $K_{p}$ are load and pressuredependent variables, respectively. Finally, parameter

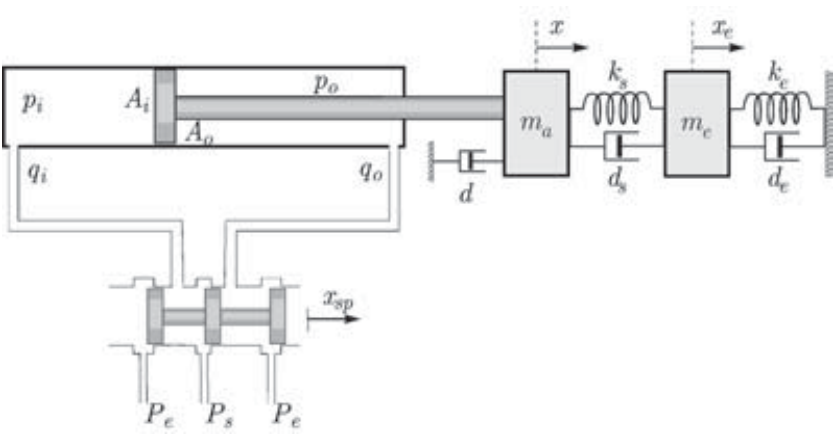

Fig. 5 Diagram of the hydraulic actuator (based on reference $[\mathbf{2 5}]$ ) 
$C$ is a constant arising from the linearization procedure around a specified operating point, defined as

$$
C=\frac{1}{\beta}\left(\frac{\bar{V}_{\mathrm{i}}+\bar{V}_{\mathrm{o}}}{2}\right)
$$

where $\bar{V}_{\mathrm{i}}$ and $\bar{V}_{\mathrm{o}}$ represent the initial volumes of hydraulic fluid trapped in the blind and the rod sides of the piston and $\beta$ is the effective bulk modulus of the fluid. The remaining variables defined in Fig. 5 are: $x$ (piston displacement), $x_{e}$ (load-mass displacement), $p_{\mathrm{i}}$ and $p_{\mathrm{o}}$ (input and output line pressures respectively), $q_{\mathrm{i}}$ and $q_{\mathrm{o}}$ (fluid flowrates into and out of the valve respectively), $p_{\mathrm{s}}$ and $p_{\mathrm{e}}$ (pump and return - exit - pressures respectively), and $x_{\mathrm{sp}}$ (spool displacement).

A list of all parameters defining the linearized transfer function is given in Table 1. For each parameter a minimum, maximum and nominal value is given. The parameters are assumed to vary independently between their corresponding extreme values.

The uncertainty in $K_{s}$ and $K_{p}$ reflects variations in the operating point (especially the non-linearity arising at the interface between positive and negative spool displacements), supply pressure, and orifice area gradient. Uncertainty in parameters $k_{e}$ and $d$ model variations in environmental stiffness and damping, while uncertainty in valve characteristics is modelled by variations in parameters $\tau$ and $k_{\mathrm{sp}}$ [25]. Variations in parameter $C$ reflect changes in the fluid bulk modules and the volumes of the fluid trapped at the sides of the actuator. All these parameters are known to affect the dynamic stability of the system.

Following reference [25], the design frequencies were chosen as $\Omega=\{0.01,0.05,0.1,0.5,15,10,50,70$, $100\} \mathrm{rad} / \mathrm{s}$. The robust tracking bounds are defined by the magnitude frequency response of the two systems

$$
B_{\mathrm{u}}(s)=\frac{s / 2.8+1}{(s / 4+1)(s / 7+1)(s / 8+1)}
$$

Table 1 Operating values and parameter ranges

\begin{tabular}{lll}
\hline Parameter & Nominal value & Range \\
\hline$k_{e}$ & $75 \mathrm{kN} / \mathrm{m}$ & $50-100$ \\
$K_{s}$ & $0.375 \mathrm{~m}^{3} / \mathrm{Pa} \mathrm{s}$ & $0.25-0.5$ \\
$K_{p}$ & $2.5 \times 10^{-12} \mathrm{~m}^{2} / \mathrm{s}$ & $0-5 \times 10^{-12}$ \\
$C$ & $1.5 \times 10^{-11} \mathrm{~m}^{3} / \mathrm{Pa}$ & $1 \times 10^{-11}-3 \times 10^{-11}$ \\
$d$ & $700 \mathrm{~N} / \mathrm{m} \mathrm{s}^{2}$ & $600-800$ \\
$m_{a}$ & $20 \mathrm{~kg}$ & $19.9-20.1$ \\
$A_{\mathrm{i}}$ & $0.00203 \mathrm{~m}^{2}$ & $0.00193-0.00213$ \\
$A_{\mathrm{o}}$ & $0.00152 \mathrm{~m}^{2}$ & $0.00144-0.00160$ \\
$k_{\mathrm{sp}}$ & $0.0012 \mathrm{~m} / \mathrm{V}$ & $0.0011-0.0013$ \\
$\tau$ & $35 \mathrm{~ms}$ & $30-40$ \\
\hline
\end{tabular}

and

$$
B_{1}(s)=\frac{1}{(s / 4.8+1)(s / 80+1)\left(s^{2} / 50+9.6 s / 50+1\right)}
$$

These were obtained by step-response figures of merit related to rise-time, percentage overshoot, and settling time (for full details refer to reference [25]). Thus the design specifications for the feedback controller $K(s)$ are

$$
\begin{aligned}
& \max _{\boldsymbol{p} \in \mathscr{P}}\left|\frac{G\left(\boldsymbol{p}, \mathrm{j} \omega_{i}\right) K\left(\mathrm{j} \omega_{i}\right)}{1+G\left(\boldsymbol{p}, \mathrm{j} \omega_{i}\right) K\left(\mathrm{j} \omega_{i}\right)}\right|_{\mathrm{dB}} \\
& \quad \leqslant\left|B_{\mathrm{u}}\left(\mathrm{j} \omega_{i}\right)\right|_{\mathrm{dB}}-\left|B_{\mathrm{i}}\left(\mathrm{j} \omega_{i}\right)\right|_{\mathrm{dB}}
\end{aligned}
$$

which should hold for all design frequencies in $\Omega$ and for every possible combination of the ten uncertain parameters varying over their respective ranges specified in Table 1. Further, it is required that the open-loop frequency response (for any permissible combination of parameters) should not enter the $M=1.4$ circle, which gives the design an approximate gain margin of $3 \mathrm{~dB}$. Finally, since for hydraulic actuators of this type the valve dead-band typically produces a steady state error in the system response [25], integral action is required from the feedback controller to eliminate the steady state error.

The uncertainty templates of the model were first obtained by using a three-point grid for each uncertain parameter (nominal, minimum, and maximum values). This resulted in $3^{10}=59049$ uncertain points in the Nichols chart for each template. To reduce the number of subsequent calculations, the convex hull of each uncertainty template was also obtained and used to derive the Horowitz bounds at each design frequency. This process introduces some measure of conservativeness to the design, as the uncertainty templates need not be convex, which in this case, however, is minimal.

The Horowitz bounds were next calculated numerically at the ten design frequencies, with a gain tolerance of $0.1 \mathrm{~dB}$ and a phase step of $1^{\circ}$. This was followed by the construction of the $\mathscr{U}$-contour, corresponding to an $M$-circle with $M=1.4$ and a high-frequency uncertainty spread of $V_{\infty}=11.03 \mathrm{~dB}$, calculated analytically from the model. The corresponding contours are shown in Fig. 6, together with the nominal frequency response of the plant (the ten design frequencies being marked with a circle). Note that only the first seven design frequencies correspond to open Horowitz templates.

The design specifications indicate that integral action must be introduced via the feedback controller. First, it was attempted to design an optimal PID 


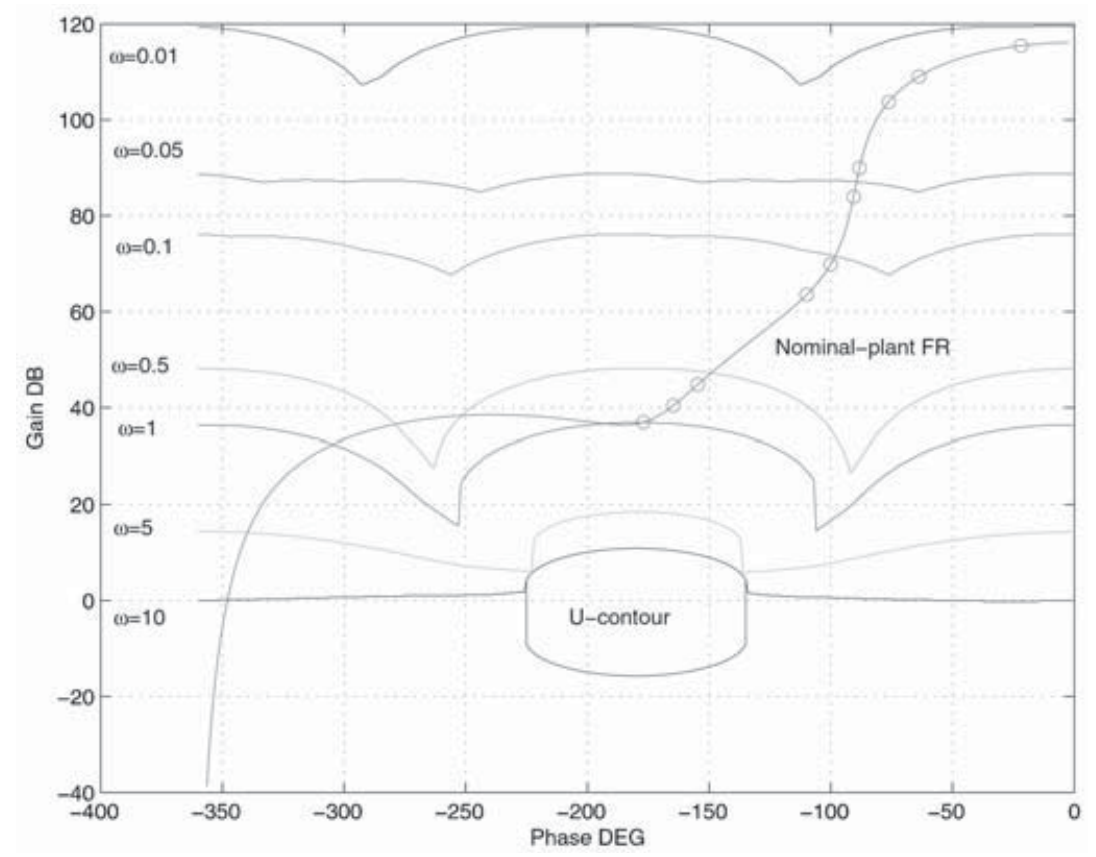

Fig. 6 Nominal-plant frequency response, Horowitz templates, and $\mathscr{U}$-contour

controller using the results of Theorem 1, which proved to be infeasible. The reason in clear from Fig. 6, which indicates that a large amount of phase advance (exceeding $90^{\circ}$ ) should be introduced in the mid-high frequency range. Thus the controller structure was modified as

$$
K_{1}(s)=\frac{k_{1}+k_{2} s+k_{3} s^{2}}{s(s / 130+1)}
$$

The $s$-term in the denominator provides the required integral action, while the numerator is a $\mathrm{PDD}^{2}$ (proportional derivative-double derivative) term providing sufficient phase advance (up to $180^{\circ}$ at high frequencies). The additional pole at $s=-130$ was introduced to ensure that the controller is proper. The optimal location of this additional pole could be optimized using this method, although its effects in this case are minimal. Next, the denominator term of $K_{1}(s)$ was absorbed by the nominal plant, and the three parameters $k_{1}, k_{2}$, and $k_{3}$ were optimized using the algorithm of section 3 and the results of Theorem 1'. The cost function chosen for optimization was the open-loop asymptotic gain (controlled by $k_{3}$ ). The new optimization problem proved feasible and resulted in an optimal $\mathrm{PDD}^{2}$-controller with

$$
k_{1}^{*}=0.004, \quad k_{2}^{*}=0.002, \quad k_{3}^{*}=4.9778 \times 10^{-5}
$$

The resulting open-loop system is shown in Fig. 7.

It can be seen that the design specifications at all ten design frequencies are satisfied, the first seven points lying on or above the corresponding templates, the last three (high frequencies corresponding to the closed Horowitz contours) lying outside or on the $\mathscr{U}$-template. It may be seen, however, that the nominal frequency response penetrates the $\mathscr{U}$-contour between the two consecutive design frequencies $\omega=10$ and $\omega=50 \mathrm{rad} / \mathrm{s}$. This is a common problem with QFT design which is based on a discrete set of design frequencies. A typical remedy is to define a more dense set of design frequencies or tighten the specifications. Here a simpler technique was followed by adding an additional first-order lag term to the controller, to modify the open-loop response in the offending frequency range $10 \leqslant \omega \leqslant$ $50 \mathrm{rad} / \mathrm{s}$. The overall controller is

$$
K(s)=\frac{\left(0.0004+0.002 s+4.9778 \times 10^{-5} s^{2}\right)(0.06231 s+1)}{s(s / 130+1)(0.1295 s+1)}
$$

The corresponding open-loop response is shown in Fig. 8. The nominal open-loop system has a crossover frequency of $16.91 \mathrm{rad} / \mathrm{s}$ and a $3 \mathrm{~dB}$ closed-loop bandwidth equal to $28.21 \mathrm{rad} / \mathrm{s}$.

The controller designed using the proposed optimization method is remarkably similar to the one designed manually in reference [25] (the controller in reference [25] was also validated experimentally). This suggests that the design specifications in this case are tight. Moreover, the similarity can be further illustrated in Figs 9 and 10, comparing the magnitude and phase responses of both the optimized and 


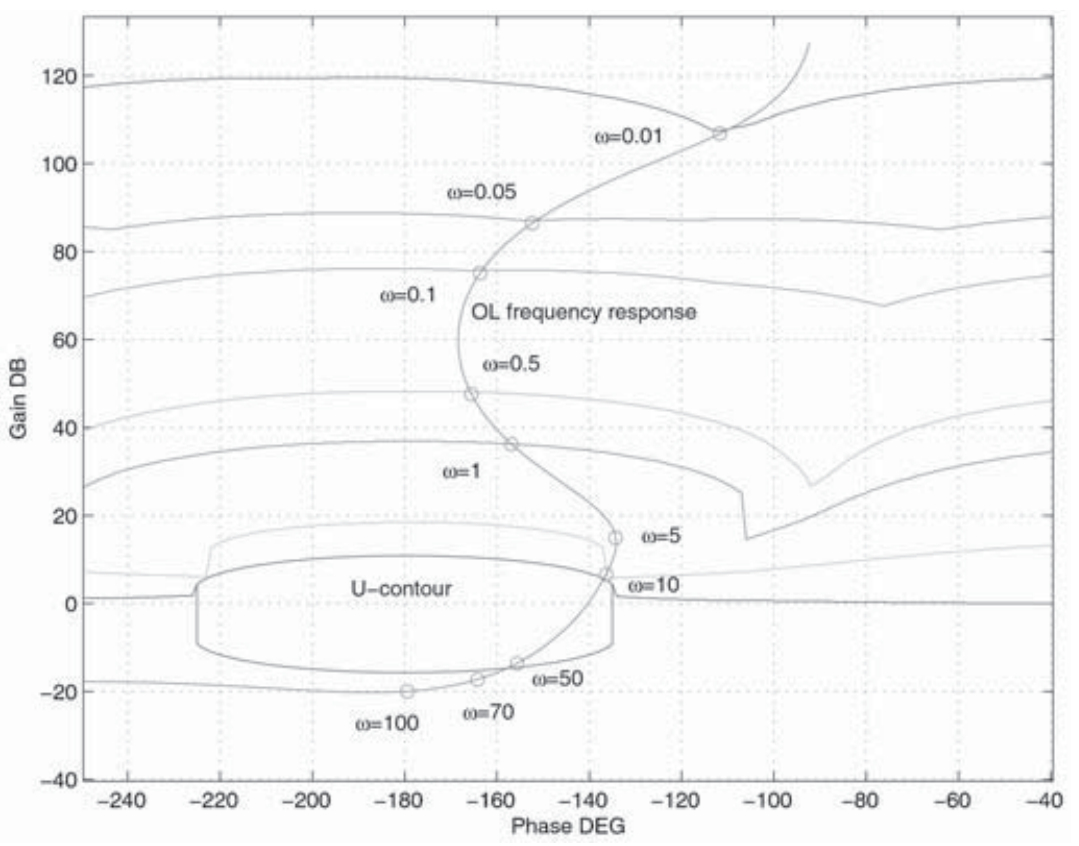

Fig. 7 Nominal open-loop response with the $\mathrm{PDD}^{2}$ optimal controller

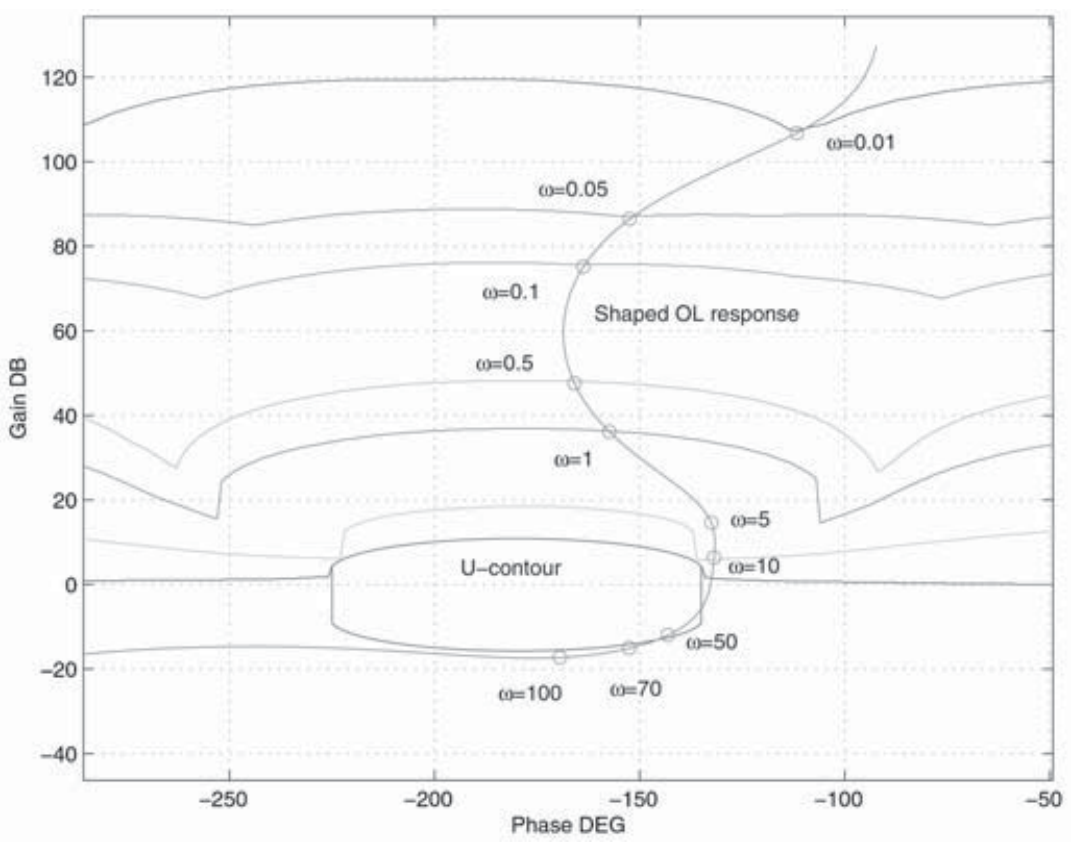

Fig. 8 Nominal open-loop response with the modified $\mathrm{PDD}^{2}$ optimal controller

manually designed controllers. The optimized controller is able to meet the robust performance specifications with less gain at low frequencies (of around $4 \mathrm{~dB}$ ) but has a lower roll-off rate at high frequencies. The phase responses of the two controllers are also similar; they both inject phase lag at low frequencies (due to their integral action) and provide maximum phase advance in the range $50-80 \mathrm{rad} / \mathrm{s}$ to improve the robust stability margins of the two designs. This illustrates the usefulness of this method for designing controllers for practical systems.

\subsection{Pre-filter design}

The ultimate step of the QFT design is to design a pre-filter. Here the following procedure was used. First, the magnitude frequency responses of $3^{5}=243$ 


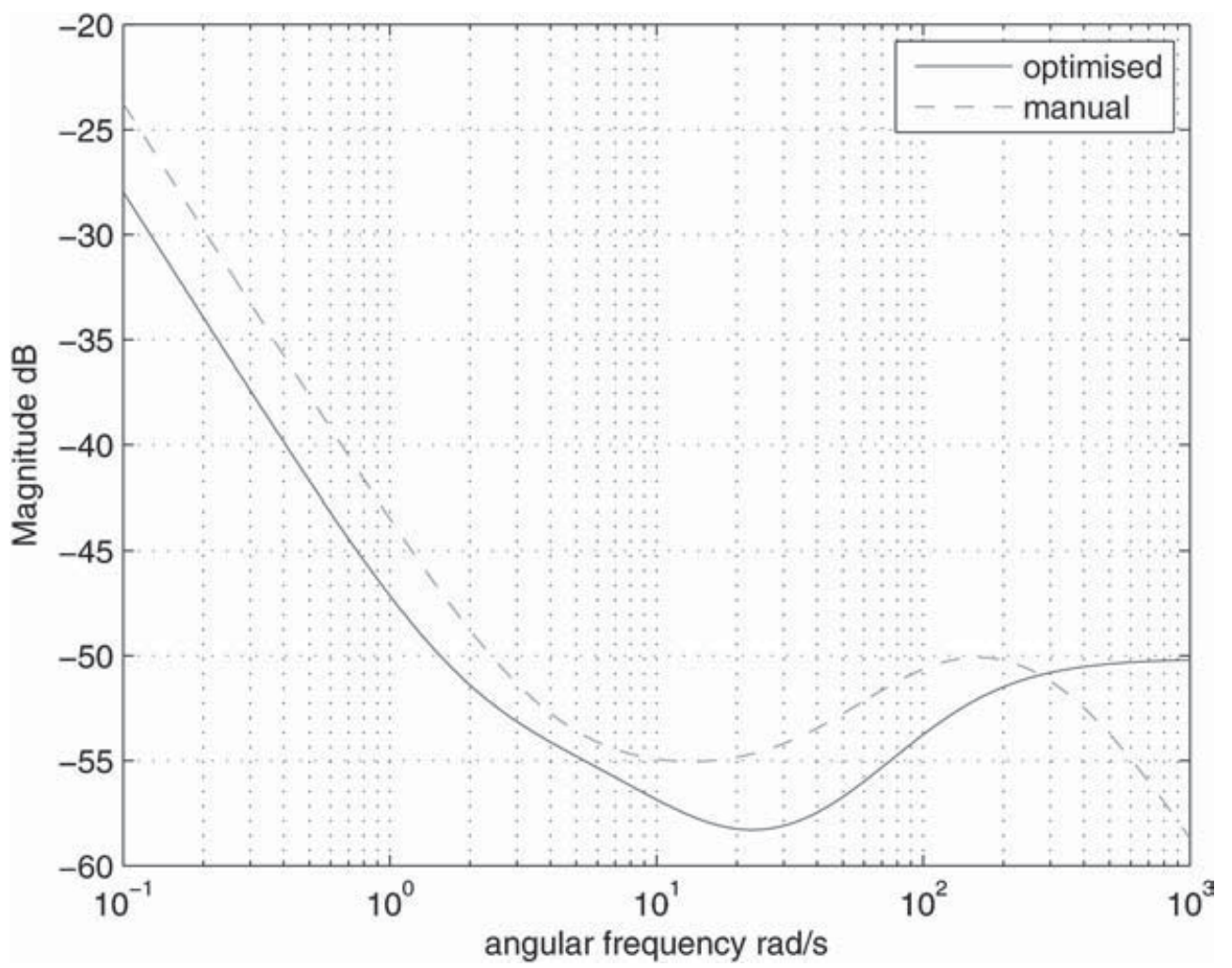

Fig. 9 Magnitude response comparison of the proposed optimized and manual-designed (from reference [25]) controllers

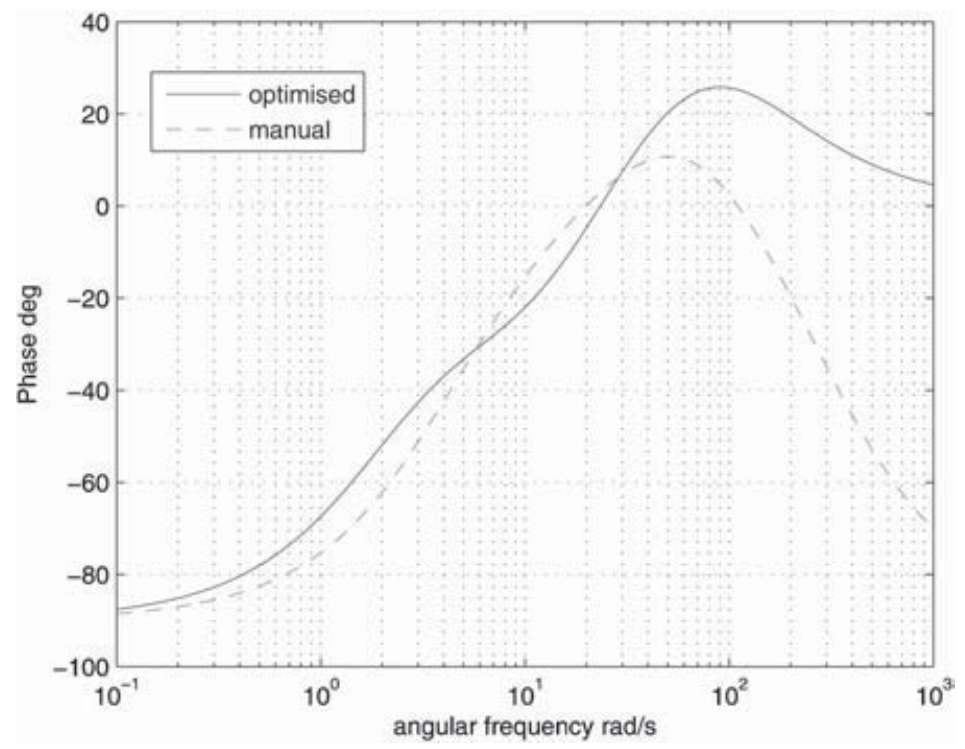

Fig. 10 Phase response comparison of the proposed optimized and manual-designed (from reference [25]) controllers

open-loop uncertain systems were plotted (see Fig. 11). These correspond to the five more important parameters (in terms of the uncertainty template spread), the remaining five parameters being fixed to their nominal value. Next, the maximum and minimum gains were recorded at the ten design frequencies. The values obtained are summarized in Tables 2 and 3.

As expected, the spread in the closed-loop gain is within the required tolerances; thus all responses can be brought between the specified lower and upper bounds by designing a pre-filter that essentially 


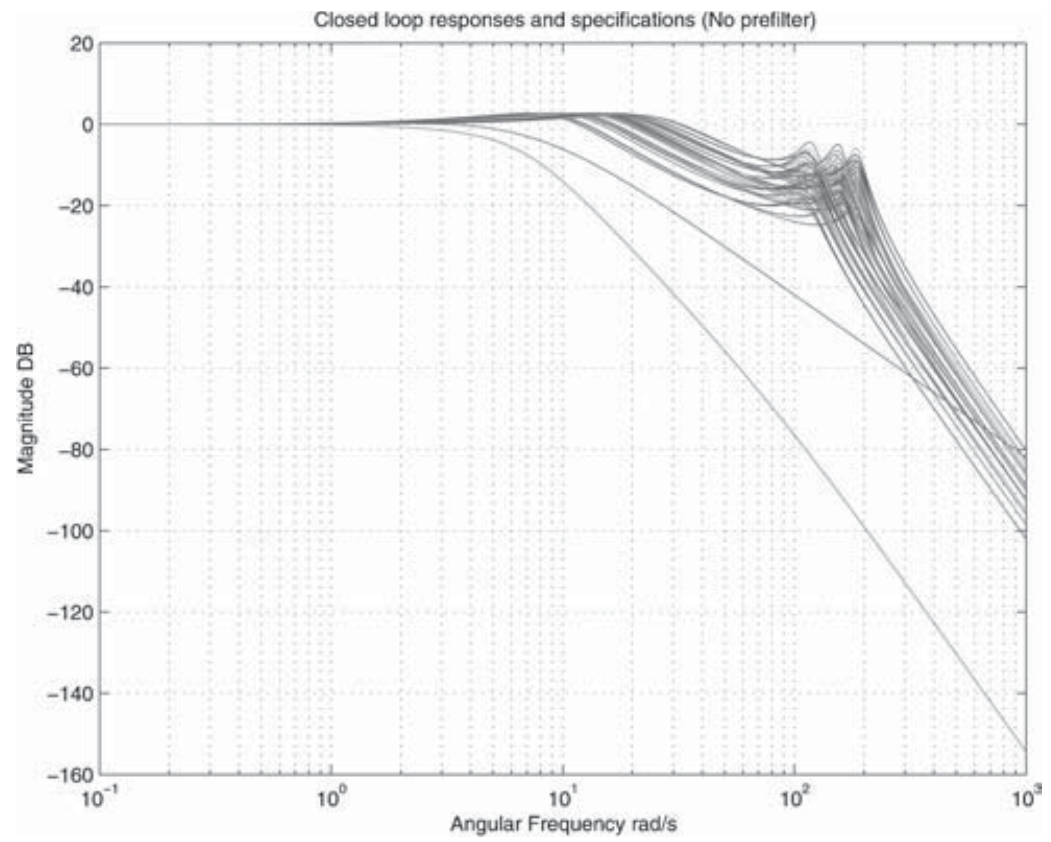

Fig. 11 Closed-loop frequency responses - no pre-filter

Table 2 Closed-loop specifications

\begin{tabular}{ccc}
\hline Frequency $(\mathrm{rad} / \mathrm{s})$ & Upper bound $(\mathrm{dB})$ & Lower bound $(\mathrm{dB})$ \\
\hline 0.01 & 0.0000 & 0.0000 \\
0.05 & 0.0003 & -0.0004 \\
0.10 & 0.0013 & -0.0018 \\
0.50 & 0.0300 & -0.0437 \\
1.00 & 0.1030 & -0.1733 \\
5.00 & -1.0882 & -3.8959 \\
10.00 & -6.1353 & -14.0514 \\
50.00 & -30.1052 & -55.7940 \\
70.00 & -35.8489 & -65.5845 \\
100.00 & -41.9907 & -76.4891 \\
\hline
\end{tabular}

Table 3 Closed-loop minimum and maximum gains

\begin{tabular}{lcc}
\hline Frequency $(\mathrm{rad} / \mathrm{s})$ & Maximum gain $(\mathrm{dB})$ & Minimum gain $(\mathrm{dB})$ \\
\hline 0.01 & 0.0000 & 0.0000 \\
0.05 & 0.0008 & 0.0002 \\
0.10 & 0.0032 & 0.0007 \\
0.50 & 0.0777 & 0.0177 \\
1.00 & 0.2796 & 0.0631 \\
5.00 & 2.2479 & 0.5656 \\
10.00 & 2.7065 & 1.3864 \\
50.00 & -4.7396 & -17.7778 \\
70.00 & -8.3369 & -21.1456 \\
100.00 & -7.1198 & -23.9876 \\
\hline
\end{tabular}

provides frequency-dependent scaling. In this case, the pre-filter must provide adequate attenuation at high frequencies.

The filter was designed via an optimization procedure based on linear programming. First, the difference of the maximum gain from the upper bound was recorded at the design frequencies, together with the difference of the minimum gain from the lower frequency bound. The two differences were then averaged and this defined the required attenuation of the pre-filter (at the ten design frequencies). This procedure was followed in order to bring the responses in the middle of the specified region in the Bode diagram. With the magnitude frequency response of the filter specified at the ten design frequencies, the next task was to fit a stable rational function to approximate the response. Various techniques (e.g. least-squares) can be used for this purpose, but the one that was followed was based on MATLAB's routine fitmaglp.m (from the $\mu$-control toolbox [27]), in which fitting can be performed interactively over various filter orders, while the results are graphically displayed (target and achieved frequency response). The function formulates the problem as a linear programme using weights to

Table 4 Target and achieved pre-filter gains

\begin{tabular}{lcc}
\hline Frequency $(\mathrm{rad} / \mathrm{s})$ & Target gain $(\mathrm{dB})$ & Achieved gain $(\mathrm{dB})$ \\
\hline 0.01 & 0.0000 & 0.0000 \\
0.05 & -0.0005 & -0.0006 \\
0.10 & -0.0021 & -0.0022 \\
0.50 & -0.0528 & -0.0546 \\
1.00 & -0.2120 & -0.2065 \\
5.00 & -4.8403 & -3.8989 \\
10.00 & -12.2030 & -12.1397 \\
50.00 & -31.8440 & -31.6909 \\
70.00 & -36.7536 & -35.9755 \\
100.00 & -43.9554 & -43.6862 \\
\hline
\end{tabular}


emphasize the fit at the required frequency ranges. In this example equal weights were used for all (ten) frequencies. A filter order equal to three was found to give a good compromise between accuracy and complexity. The transfer function of the filter was obtained as

$$
F(s)=\frac{(1+s / 16.25)[1+s /(20.08 \pm \mathrm{j} 145.74)]}{(1+s / 104.36)[1+s /(4.52 \pm \mathrm{j} 2.09)]}
$$

so that $F(s)$ is both stable and minimum phase (as guaranteed by this method). The specified and achieved responses of the filter at the design frequencies are summarized in Table 4.

The closed-loop responses of the system (with the pre-filter) are shown in Fig. 12. As expected, these are all contained within the specified upper and lower bounds. The $3^{5}$ (unit) step responses of the system are finally shown in Fig. 13.

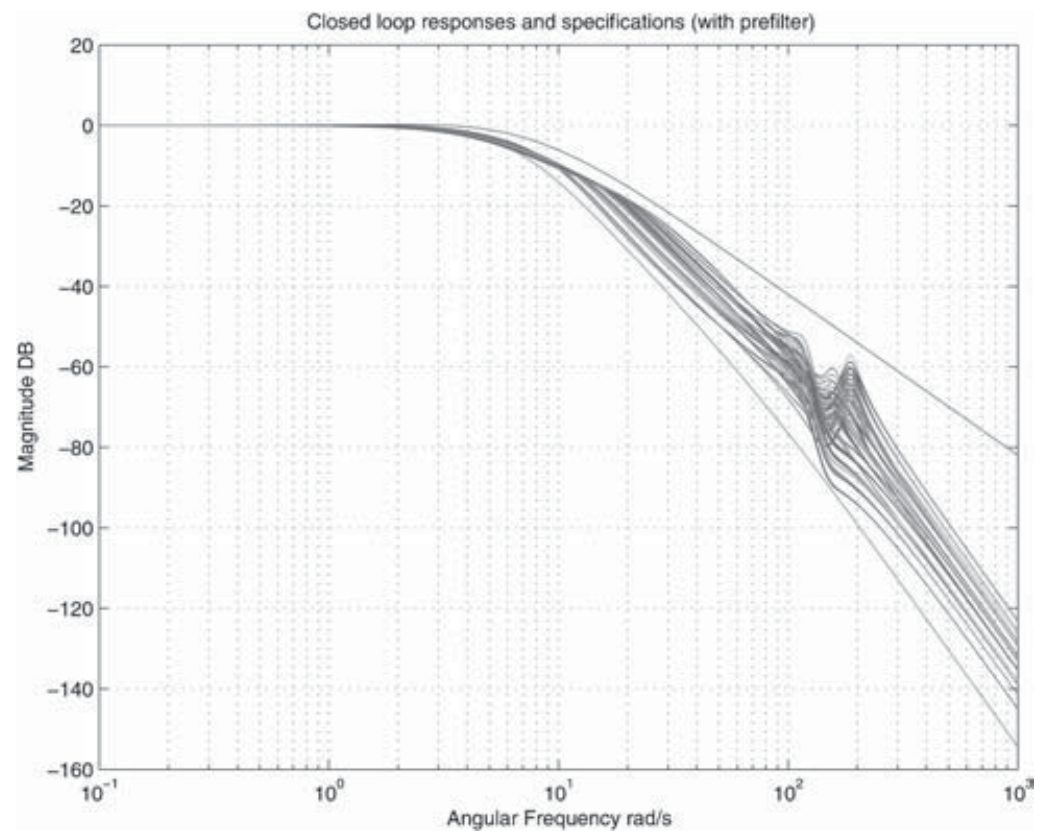

Fig. 12 Closed-loop frequency responses - with pre-filter

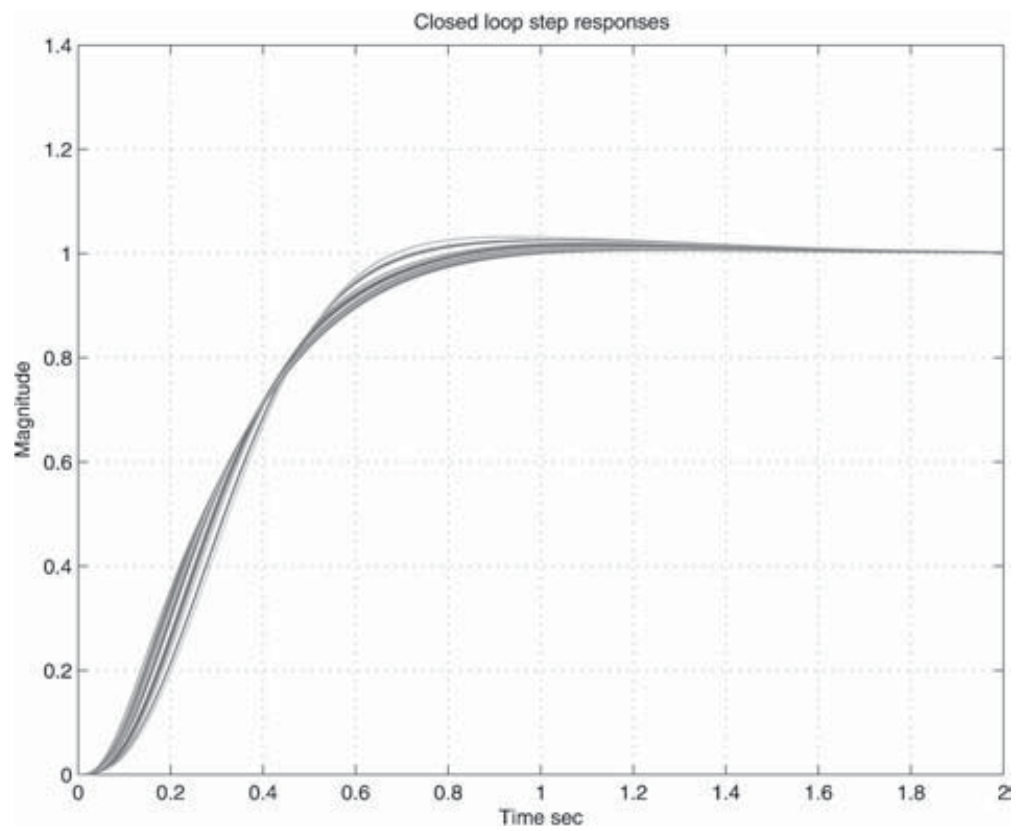

Fig. 13 Closed-loop step responses - with pre-filter 


\section{CONCLUSIONS}

An algorithm for the control design of highly uncertain systems has been outlined. The algorithm is simple, straightforward to implement, and can be used to automate the loop-shaping step of the QFT design procedure. It can be used to design robust-performance optimal controllers of a simple structure (PID, phase-lead/lag, second order), or more complex controllers involving arbitrary interconnections of these structures. The design algorithm has been illustrated with a design example of a linearized hydraulic actuator model interacting with an uncertain environment [25]. Extensions of the method to multivariable systems are possible using the standard QFT approach $[\mathbf{1}, \mathbf{2 8}]$.

\section{REFERENCES}

1 Nandakumar, R., Halikias, G. D., and Zolotas, A. An optimisation algorithm for designing fixed-structure controllers using the QFT method. IEEE CCA02CACSD, Glasgow, September 2002.

2 Yaniv, O. and Horowitz, I. M. A quantitative design method for MIMO linear feedback systems having uncertain plants. Int. J. Control, 1986, 43, 401-421.

3 Bishop, R. and Dorf, R. Modern control systems, 9th edition, 2001 (Pearson/Prentice-Hall, NJ, USA).

4 Ballance, D. I. and Gawthrop, P. J. Control system design via a quantitative feedback approach. In Proceedings of the IEE Conference Control-91, 1991, Heriot-Watt University, Edinburgh, Vol. 1, pp. $476-480$.

5 Horowitz, I. M. Synthesis of linear systems, 1973 (Academic Press, New York).

6 Horowitz, I. M. and Sidi, M. Synthesis of linear systems with large plant ignorance for prescribed time-domain tolerances. Int. J. Control, 1972, 16, 287-309.

7 Horowitz, I. M. and Sidi, M. Optimum synthesis of non-minimum phase systems with plant uncertainty. 1978, Int. J. Control, 27, 361-386.

8 Chen, W. and Ballance, D. J. QFT design for uncertain non-minimum phase and unstable plants. Preprint, Centre for Systems and Control, Department of Mechanical Engineering, University of Glasgow, July 1997.

9 Chen, W. and Ballance, D. J. Stability analysis on the Nichols chart and its application in QFT. Preprint, Centre for Systems and Control, Department of Mechanical Engineering, University of Glasgow, August 1997.

10 Rodriguez, J. M., Chait, Y., and Hollot, C. V. A new algorithm for computing QFT bounds. Trans. ASME, J. Dynamic Systems, Measmt and Control, 1997, 119, 548-552.

11 Braatz, R. D. A reconciliation between quantitative feedback theory and robust multivariable control.
In Proceedings of the American Control Conference, Baltimore, Maryland, 1994, pp. 3374-3379.

12 Bryant, G. F. and Halikias, G. D. Optimum loopshaping for systems with large parameter uncertainty via linear programming. Int. J. Control, 1995, 62(3), 557-568.

13 Thompson, D. F. and Nwokah, O. D. I. Analytic loop shaping methods in quantitative feedback theory. Trans. ASME, J. Dynamic Systems, Meamt and Control, 1994, 116, 169-177.

14 Ukpai, U. I. and Jayasuriya, S. Gain-bandwidth optimization of PID controllers. In Proceedings of the Americal Control Conference, Anchorage, Alaska, 2002, pp. 4979-4984.

15 Zolotas, A. C. and Halikias, G. D. Optimal design of PID controllers using the QFT method. IEE Proc.Control Theory Applic., November 1999, 146(6).

16 Gera, A. and Horowitz, I. Optimization of the loop transfer function. Int. J. Control, 1980, 31, 389-398.

17 Chait, Y., Chen, Q., and Hollot, C. V. Automatic loop-shaping of QFT controllers via linear programing. Trans. ASME J. Dynamic Systems, Measmt and Contro1, 1999, 121, 351-357.

18 Yaniv, O. and Nagurka, M. Robust PI controller design satisfying sensitivity and uncertainty specifications. IEEE Autom. Control, 2003, 48(11), 2069-2072.

19 Yaniv, O. and Nagurka, M. Design of PID controllers satisfying gain margins and sensitivity constraints on a set of plants. Automatica, 2004, 40(1), 111-116.

20 D'Azzo, J. and Houpis, C. Feedback control systems analysis and synthesis, 1998 (Prentice-Hall, Englewood Cliffs, New Jersey).

21 Panagopoulos, H., Astrom, K. J., and Hagglund, T. Design of PI controllers based on non-convex optimization. Automatica, 1998, 34(5), 585-601.

22 Nhowkah, O. D. I., Rordgren, R. E., and Grewal, G. S. Optimization of loop transmission functions in SISO uncertain feedback systems. In Proceedings of the American Control Conference Baltimore, Maryland, 1994, pp. 365-373.

23 Franson, C. M., Lennartson, B., Wik, T., Holnstrom, K., Saunders, M., and Gutman, P. 0. Global controller optimization using Horowitz bounds. In IFAC World Congress, Barcelona, Spain, 2002.

24 Astrom, K. J. and Hagglund, T. The future of PID control. Control Engng Practice, 2001, 9, 1163-1175.

25 Niksefat, N. and Sepehri, N. Designing robust force control of hydraulic actuators despite system and environmental uncertainties. IEEE Control Systems Mag., April 2001.

26 Niksefat, N. and Sepehri, N. Robust force controller design for an electro-hydraulic actuator based on a nonlinear model. In Proceedings of the IEEE International Conference on Robotics and automation, Detroit, Michigan, 1999, pp. 200-206.

27 Balas, G. J., Doyle, J. C., Glover, K., Packard, A., and Smith, R. $\mu$-Analysis and synthesis toolbox for use with Matlab, 2001 (MathWorks Inc., Natick, Massachusetts).

28 Maciejowski, J. M. Multivariable feedback design, 1989 (Addison-Wesley, Reading, Massachusetts). 